\title{
Spatial particle correlations in light nuclei. I Two-particle systems
}

\author{
P. Mei and P. Van Isacker \\ GANIL, CEA/DSM-CNRS/IN2P3, BP 55027, F-14076 Caen Cedex 5, France
}

\begin{abstract}
Expressions for spatial two-particle correlations in an $L S$-coupled basis of the harmonic oscillator are used to display the probability distribution of two identical nucleons as a function of their relative distance and their distance from the center of the nucleus. It is shown that a two-nucleon state in the $p$ shell with total orbital angular momentum $L=0$ and total spin $S=0$ contains a di-neutron and a cigarlike component with equal probability. This result can also be proven analytically with the use of angular correlation functions. Scattering of the nucleons from the $p$ shell to other shells leads to the enhancement of the di-neutron configuration. A semi-quantitative application to ${ }^{6} \mathrm{He}$ is presented which shows that the probability of the di-neutron configuration in the ground state is of the order of $60 \%$. The longterm goal of this work is to obtain a geometric insight into the properties of nuclei with several nucleons in a valence shell.
\end{abstract}

Key words: few-body systems, shell model, neutron distributions PACS: 21.45.+v, 21.60.Cs, 21.10.Gv

\section{Introduction}

The radius of nuclei close to the stability line grows on average as the power $1 / 3$ of the mass number $A$. It came therefore as a surprise that for a nucleus as light as ${ }^{11} \mathrm{Li}$ an unusually large value for this quantity was deduced from interaction-cross-section measurements [1]. Subsequently, it was realized that this exceptional spatial extension is due to the weak binding of the two outer neutrons to the ${ }^{9} \mathrm{Li}$ core; the term 'halo' was coined to indicate the phenomenon of one or two particles wandering into a classically forbidden region around a tightly bound core. After the introduction of this concept in the study of light nuclear systems in the 1980s, it migrated to atomic and molecular physics, prompting a generic treatment of halo properties in diverse systems [2]. Meanwhile, in nuclear physics, other examples of halo nuclei were 
found, among which the isotopes ${ }^{6} \mathrm{He}$ and ${ }^{8} \mathrm{He}[3]$, the focus of the first two of the present series of papers.

These experimental studies of neutron-rich light nuclei prompted theoretical work using a variety of approaches, as reviewed by Zhukov et al. [4]. In the case of ${ }^{6} \mathrm{He}$ most of the calculations were of a three-body character (the $\alpha$ particle and two neutrons) and all employed rather sophisticated techniques such as an expansion in hyperspherical harmonics, the coordinate-space Faddeev approach or the two-particle Green's function method. In spite of their complexity these calculations gave rise to a simple geometric picture of ${ }^{6} \mathrm{He}$, with a ground state divided between a 'di-neutron' and a 'cigar-like' configuration (see Fig. 3 of Ref. [4]).

In this series of papers we explore the geometry of few-particle systems starting from the nuclear shell model formulated in a harmonic-oscillator basis. With applications to the nuclei ${ }^{6} \mathrm{He}$ and ${ }^{8} \mathrm{He}$ in mind, we concentrate in papers I and II on systems with two and four identical particles, respectively. In these first two papers the appropriate background and algorithms are developed, necessary to probe the geometric structure of systems of non-identical particles which will be the subject of the third in the series. Our approach is basic and does not require techniques that go beyond elementary quantum mechanics. It uses in particular the concept of two-particle correlation function which is recalled in Sect. 2. Within this simple approach it can be shown (Sect. 3) that the di-neutron and cigar-like configurations mentioned above are an inevitable consequence of the geometry of the $p$ shell, to which the effect of correlations should be added. This property can be derived analytically with use of the angular correlation function between the two particles, which will be the more appropriate formulation for the generalization toward more than two particles. A semi-quantitative application of this approach to ${ }^{6} \mathrm{He}$ is considered in Sect. 4. In Sect. 5 the conclusions and perspectives of this work are formulated.

\section{Two-particle correlation functions}

The relevant physical observables that determine the spatial structure of two valence nucleons outside an inert core, such as in ${ }^{6} \mathrm{He}$, are the relative distance between the two nucleons and the distance between the the center of mass of the closed-shell core and that of the two nucleons. These observables can be probed by what will be called two-particle correlation functions. A two-particle correlation function is the expectation value of $\delta\left(\bar{r}-\bar{r}_{12}\right)$, where $\bar{r}_{12} \equiv \bar{r}_{1}-\bar{r}_{2}$ is the difference between the position vectors of the two particles, $\bar{r}_{1}$ and $\bar{r}_{2}$, and $\bar{r}$ is an arbitrary vector. The expectation value of this operator, multiplied with the appropriate volume element, measures the probability of finding the two particles separated by $\bar{r}$. Usually one is only interested in this probability 
as a function of the distance $r_{12} \equiv\left|\bar{r}_{1}-\bar{r}_{2}\right|$ between the two particles which then involves the operator $\delta\left(r-r_{12}\right)$. Also, it is often useful to probe in addition the distance of the two particles from the center of the nucleus and this can be achieved by calculating the expectation value of $\delta\left(r-r_{12}\right) \delta\left(R-R_{12}\right)$, where $R_{12} \equiv\left|\bar{r}_{1}+\bar{r}_{2}\right| / 2$ is the radial coordinate of the center of mass of the two particles.

These notions can be applied to any quantum-mechanical many-body state by defining the correlation functions

$$
\begin{aligned}
\mathcal{C}_{\alpha \alpha^{\prime}}(\bar{r}) & \equiv\left\langle\alpha\left|\sum_{i<j} \delta\left(\bar{r}-\bar{r}_{i j}\right)\right| \alpha^{\prime}\right\rangle, \\
\mathcal{C}_{\alpha \alpha^{\prime}}(r) & \equiv\left\langle\alpha\left|\sum_{i<j} \delta\left(r-r_{i j}\right)\right| \alpha^{\prime}\right\rangle, \\
\mathcal{C}_{\alpha \alpha^{\prime}}(r, R) & \equiv\left\langle\alpha\left|\sum_{i<j} \delta\left(r-r_{i j}\right) \delta\left(R-R_{i j}\right)\right| \alpha^{\prime}\right\rangle,
\end{aligned}
$$

where the sum is over all particles in the $n$-particle states $|\alpha\rangle$ and $\left|\alpha^{\prime}\right\rangle$, characterized by a set of labels collectively denoted as $\alpha$ or $\alpha^{\prime}$, and in general comprising the total angular momentum $J$ and its projection $M_{J}$. It often will be convenient to refer to all correlation functions (1) simultaneously, in which case the notation $\mathcal{C}_{\alpha \alpha^{\prime}}$ will be used. The interpretation of $\mathcal{C}_{\alpha \alpha^{\prime}}$ as a probability distribution is valid in the diagonal case, $\alpha=\alpha^{\prime}$, but expressions for nondiagonal matrix elements are needed as well in the following. As the operators $\delta\left(r-r_{12}\right)$ and $\delta\left(r-r_{12}\right) \delta\left(R-R_{12}\right)$ are scalar under rotations, their matrix elements are diagonal in $J$ and independent of $M_{J}$. This is not the case for the operator $\delta\left(\bar{r}-\bar{r}_{12}\right)$ which therefore leads to more complicated expressions for its matrix elements. Correlation functions satisfy the normalization conditions

$$
\begin{aligned}
& \int_{\Re^{3}} \mathcal{C}_{\alpha}(\bar{r}) d \bar{r}=\frac{n(n-1)}{2}, \\
& 4 \pi \int_{0}^{+\infty} \mathcal{C}_{\alpha}(r) r^{2} d r=\frac{n(n-1)}{2}, \\
& 16 \pi^{2} \int_{0}^{+\infty} r^{2} d r \int_{0}^{+\infty} \mathcal{C}_{\alpha}(r, R) R^{2} d R=\frac{n(n-1)}{2},
\end{aligned}
$$

where the notation $\mathcal{C}_{\alpha} \equiv \mathcal{C}_{\alpha \alpha}$ is used, as well as the orthogonality conditions (with $\alpha \neq \alpha^{\prime}$ )

$$
\begin{aligned}
& \int_{\Re^{3}} \mathcal{C}_{\alpha \alpha^{\prime}}(\bar{r}) d \bar{r}=0, \\
& \int_{0}^{+\infty} \mathcal{C}_{\alpha \alpha^{\prime}}(r) r^{2} d r=0, \\
& \int_{0}^{+\infty} r^{2} d r \int_{0}^{+\infty} \mathcal{C}_{\alpha \alpha^{\prime}}(r, R) R^{2} d R=0 .
\end{aligned}
$$


These are generic conditions, valid for any of the correlation functions that will be derived below.

\section{Two-particle correlations in $L S$ coupling}

Most of the calculations reported in this paper are carried in the $L S$-coupling scheme. Although nuclear physicists, after the introduction of the spin-orbit interaction by Mayer [5] and by Jensen et al. [6], have been converted to adopting the $j j$-coupling scheme, the alternative $L S$ coupling still has arguments in favor of it. One argument is that it allows a clear separation between spin and spatial degrees of freedom. One of the basic properties of the nucleonnucleon interaction is its short-range, attractive character which favors spatial symmetry and its consequences are most easily understood in $L S$ coupling. For example, an extreme short-range interaction $\delta\left(\bar{r}_{1}-\bar{r}_{2}\right)$ has a non-vanishing two-body interaction matrix element only between the space-symmetric states with $S=0, T=1$ or $S=1, T=0$. Another argument is that realistic shellmodel interactions, such as the USD interaction for the $1 s 0 d$ shell [7] or the GX1A interaction for the $1 p 0 f$ shell [8], are more diagonal in $L S$ than they are in $j j$ coupling. Last but not least, light nuclei (in the $0 p$ shell and the beginning of $1 s 0 d$ shell), to a good approximation can be classified in $L S$ coupling [9].

Let us begin with some elementary definitions, mainly to fix our notation. A single-particle wave function is determined by its spatial part, $\phi_{n \ell m_{\ell}}(\bar{r}) \equiv$ $\phi_{n \ell m_{\ell}}(r, \theta, \phi)$, multiplied by a spinor for a spin-1/2 particle, $\chi_{ \pm 1 / 2}$. For any central potential the spatial part can be written as the product of a radial wave function $R_{n \ell}(r)$, characterized by the radial quantum number $n$ and the orbital angular momentum $\ell$, and a spherical harmonic $Y_{\ell m_{\ell}}(\theta, \phi)$, characterized by $\ell$ and its projection $m_{\ell}$. It will sometimes be convenient to denote the polar and azimuthal angles $\theta$ and $\phi$ collectively as $\Omega$, so that $Y_{\ell m_{\ell}}(\Omega) \equiv Y_{\ell m_{\ell}}(\theta, \phi)$.

A two-nucleon state in $L S$ coupling is denoted as $\left|n_{1} \ell_{1} s, n_{2} \ell_{2} s ; L M_{L} S M_{S}\right\rangle \equiv$ $\left|n_{1} \ell_{1} n_{2} \ell_{2} ; L M_{L} S M_{S}\right\rangle$. As the particles always have $s=1 / 2$, their spins are not shown explicitly; their coupled value is indicated as $S$. If two identical particles are in the same $n \ell$ shell, the values of the total orbital angular momentum $L$ and the total spin $S$ are restricted by overall antisymmetry of the wave function, that is, even $L$ for $S=0$ (spatially symmetric) and odd $L$ for $S=$ 1 (spatially antisymmetric). Since the operators $\delta\left(\bar{r}-\bar{r}_{12}\right), \delta\left(r-r_{12}\right)$ and $\delta\left(r-r_{12}\right) \delta\left(R-R_{12}\right)$ are independent of spin, the correlation functions only depend on the orbital angular momentum $L$. Note, however, that allowed values of $L$ are determined by $S$, so there exists an indirect dependence on the total spin of the two particles. Alternatively, instead of the projections $M_{L}$ and $M_{S}$, the total angular momentum $J$, obtained from the coupling of $L$ and 
$S$, and its projection $M_{J}$ can be given.

\subsection{Talmi-Moshinsky transformation}

The evaluation of the correlation functions $\mathcal{C}_{\alpha \alpha^{\prime}}$ is greatly simplified if the particles are placed in a harmonic-oscillator potential. The simplification arises because, for a harmonic oscillator, the transformation from the individual particle coordinates $\bar{r}_{1}$ and $\bar{r}_{2}$ to the relative and center-of-mass coordinates, $\bar{r}_{1}-\bar{r}_{2} \equiv \bar{r}_{12}=\left(r_{12}, \theta_{12}, \phi_{12}\right)$ and $\left(\bar{r}_{1}+\bar{r}_{2}\right) / 2 \equiv \bar{R}_{12}=\left(R_{12}, \Theta_{12}, \Phi_{12}\right)$, can be carried out by means of Talmi-Moshinsky brackets [10-12].

A Talmi-Moshinsky bracket, denoted here as $a_{n \ell \mathcal{N} \mathcal{L}, L}^{n_{1} \ell_{1} n_{2} \ell_{2}}$, is characterized by the radial and orbital quantum numbers of the two particles $\left(n_{1} \ell_{1}\right.$ and $\left.n_{2} \ell_{2}\right)$, by the similar quantum numbers pertaining to the relative and center-of-mass coordinates $(n \ell$ and $\mathcal{N} \mathcal{L}$ ), and by the coupled angular momentum $L$,

$$
\begin{aligned}
& \left\langle\bar{r}_{1}, \bar{r}_{2} \mid n_{1} \ell_{1} n_{2} \ell_{2} ; L M_{L}\right\rangle \\
& =\sum_{n \ell \mathcal{N} \mathcal{L}} a_{n \ell \mathcal{N} \mathcal{L}, L}^{n_{1} \ell_{1} n_{2} \ell_{2}}\left\langle\bar{r}_{12} / \sqrt{2}, \sqrt{2} \bar{R}_{12} \mid n \ell \mathcal{N} \mathcal{L} ; L M_{L}\right\rangle \\
& =\sum_{n \ell \mathcal{N} \mathcal{L}} a_{n \ell \mathcal{N} \mathcal{L}, L}^{n_{1} \ell_{1} n_{2} \ell_{2}}\left[\phi_{n \ell}\left(\bar{r}_{12} / \sqrt{2}\right) \times \phi_{\mathcal{N} \mathcal{L}}\left(\sqrt{2} \bar{R}_{12}\right)\right]_{M_{L}}^{(L)} \\
& =\sum_{n \ell \mathcal{N} \mathcal{L}} a_{n \ell \mathcal{N} \mathcal{L}, L}^{n_{1} \ell_{1} n_{2} \ell_{2}} R_{n \ell}\left(r_{12} / \sqrt{2}\right) R_{\mathcal{N} \mathcal{L}}\left(\sqrt{2} R_{12}\right)\left[Y_{n \ell}\left(\theta_{12}, \phi_{12}\right) \times Y_{\mathcal{N} \mathcal{L}}\left(\Theta_{12}, \Phi_{12}\right)\right]_{M_{L}}^{(L)}
\end{aligned}
$$

The number of oscillator quanta is the same before and after the transformation which translates into the condition $2 n_{1}+\ell_{1}+2 n_{2}+\ell_{2}=2 n+\ell+2 \mathcal{N}+\mathcal{L}$. In these expressions $R_{n \ell}(r)$ is the radial wave function of the harmonic oscilla-

tor [13] with $r$ expressed in units of the oscillator length $b=\sqrt{\hbar / m \omega}$, where $m$ is the nucleon mass and $\omega$ the oscillator frequency. The conventions of Barber and Cooper [14] are followed here for the definition of the Talmi-Moshinsky bracket.

\subsection{Expressions for the correlation matrix elements}

With use of the expansion

$$
\delta\left(\bar{r}-\bar{r}_{12}\right)=\frac{\delta\left(r-r_{12}\right)}{r r_{12}} \sum_{\lambda=0}^{+\infty} \sum_{\mu=-\lambda}^{+\lambda} Y_{\lambda \mu}^{*}(\theta, \phi) Y_{\lambda \mu}\left(\theta_{12}, \phi_{12}\right),
$$

the first of the correlation functions (1) can be expressed as 


$$
\begin{aligned}
{ }_{\text {nas }} & \left\langle n_{1} \ell_{1} n_{2} \ell_{2} ; L M_{L} S M_{S}\left|\delta\left(\bar{r}-\bar{r}_{12}\right)\right| n_{1}^{\prime} \ell_{1}^{\prime} n_{2}^{\prime} \ell_{2}^{\prime} ; L^{\prime} M_{L}^{\prime} S^{\prime} M_{S}^{\prime}\right\rangle_{\text {nas }} \\
= & \delta_{S S^{\prime}} \delta_{M_{S} M_{S}^{\prime}}(-)^{L+L^{\prime}-M_{L}}\left[\frac{(2 L+1)\left(2 L^{\prime}+1\right)}{32 \pi}\right]^{1 / 2} \\
& \times \sum_{\lambda=0}^{+\infty} \sum_{\mu=-\lambda}^{+\lambda}(-)^{\lambda} \sqrt{2 \lambda+1}\left(\begin{array}{ccc}
L & \lambda & L^{\prime} \\
-M_{L} & \mu & M_{L}^{\prime}
\end{array}\right) Y_{\lambda \mu}^{*}(\theta, \phi) \\
& \times \sum_{n \ell n^{\prime} \ell^{\prime} \mathcal{N} \mathcal{L}}(-)^{\mathcal{L}} \sqrt{(2 \ell+1)\left(2 \ell^{\prime}+1\right)} a_{n \ell \mathcal{N} \mathcal{L}, L}^{n_{1} \ell_{1} n_{2} \ell_{2}} \\
\quad & a_{n^{\prime} \ell^{\prime} \mathcal{N} \mathcal{L}, L^{\prime}}^{n^{\prime} \ell^{\prime} n_{2}^{\prime} \ell_{2}^{\prime}} \\
& \times\left(\begin{array}{ccc}
\ell & \lambda & \ell^{\prime} \\
0 & 0 & 0
\end{array}\right)\left\{\begin{array}{ccc}
\ell & L & \mathcal{L} \\
L^{\prime} & \ell^{\prime} & \lambda
\end{array}\right\} R_{n \ell}(r / \sqrt{2}) R_{n^{\prime} \ell^{\prime}}(r / \sqrt{2}),
\end{aligned}
$$

where the symbols in round and curly brackets are Wigner $3 j$ symbols and Racah coefficients, respectively [13]. Furthermore, with use of the property that the number of quanta is conserved by the Talmi-Moshinsky bracket, the following expressions are derived for the two other correlations functions:

$$
\begin{aligned}
\text { nas } & \left\langle n_{1} \ell_{1} n_{2} \ell_{2} ; L M_{L} S M_{S}\left|\delta\left(r-r_{12}\right)\right| n_{1}^{\prime} \ell_{1}^{\prime} n_{2}^{\prime} \ell_{2}^{\prime} ; L^{\prime} M_{L}^{\prime} S^{\prime} M_{S}^{\prime}\right\rangle_{\text {nas }} \\
= & \delta_{L L^{\prime}} \delta_{M_{L} M_{L}^{\prime}} \delta_{S S^{\prime}} \delta_{M_{S} M_{S}^{\prime}}^{\prime} \\
& \times \frac{1}{\sqrt{8}} r^{2} \sum_{n n^{\prime} \ell \mathcal{N} \mathcal{L}} a_{n \ell \mathcal{N} \mathcal{L}, L}^{n_{1} \ell_{1} n_{2} \ell_{2}} a_{n^{\prime} \ell \mathcal{N} \mathcal{L}, L}^{n_{1}^{\prime} \ell_{1}^{\prime} n_{2}^{\prime} \ell_{2}^{\prime}} R_{n \ell}(r / \sqrt{2}) R_{n^{\prime} \ell}(r / \sqrt{2}),
\end{aligned}
$$

and

$$
\begin{gathered}
{ }_{\operatorname{nas}}\left\langle n_{1} \ell_{1} n_{2} \ell_{2} ; L M_{L} S M_{S}\left|\delta\left(r-r_{12}\right) \delta\left(R-R_{12}\right)\right| n_{1}^{\prime} \ell_{1}^{\prime} n_{2}^{\prime} \ell_{2}^{\prime} ; L^{\prime} M_{L^{\prime}}^{\prime} S^{\prime} M_{S}^{\prime}\right\rangle_{\text {nas }} \\
=\delta_{L L^{\prime}} \delta_{M_{L} M_{L}^{\prime}} \delta_{S S^{\prime}} \delta_{M_{S} M_{S}^{\prime}} r^{2} R^{2} \sum_{n n^{\prime} \ell \mathcal{N} \mathcal{N}^{\prime} \mathcal{L}} a_{n \ell \mathcal{N} \mathcal{L}, L}^{n_{1} \ell_{1} n_{2} \ell_{2}} a_{n_{1}^{\prime} \ell \mathcal{N}^{\prime} \mathcal{L}, L}^{n_{1}^{\prime} \ell_{1}^{\prime} n_{2}^{\prime}{ }_{2}^{\prime}} \\
\times R_{n \ell}(r / \sqrt{2}) R_{n^{\prime} \ell}(r / \sqrt{2}) R_{\mathcal{N} \mathcal{L}}(\sqrt{2} R) R_{\mathcal{N}^{\prime} \mathcal{L}}(\sqrt{2} R) .
\end{gathered}
$$

Since the total number of oscillator quanta is conserved in the Talmi-Moshinsky bracket, it follows that $\ell_{1}+\ell_{2}$ and $\ell_{1}^{\prime}+\ell_{2}^{\prime}$ must have the same parity in the matrix elements (7) and (8), otherwise they vanish.

The expressions (6), (7) and (8) are valid if particle 1 is in orbit $n_{1} \ell_{1}\left(n_{1}^{\prime} \ell_{1}^{\prime}\right)$ and particle 2 in orbit $n_{2} \ell_{2}\left(n_{2}^{\prime} \ell_{2}^{\prime}\right)$, that is, for non-antisymmetric states in bra and ket. For identical particles states must be antisymmetric and these will be denoted as $\left|n_{1} \ell_{1} n_{2} \ell_{2} ; L M_{L} S M_{S}\right\rangle$. One has the relation

$$
\begin{aligned}
& \left|n_{1} \ell_{1} n_{2} \ell_{2} ; L M_{L} S M_{S}\right\rangle \\
& =\frac{\left|n_{1} \ell_{1} n_{2} \ell_{2} ; L M_{L} S M_{S}\right\rangle_{\mathrm{nas}}+(-)^{\ell_{1}+\ell_{2}-L-S}\left|n_{2} \ell_{2} n_{1} \ell_{1} ; L M_{L} S M_{S}\right\rangle_{\mathrm{nas}}}{\sqrt{2\left(1+\delta_{n_{1} n_{2}} \delta_{\ell_{1} \ell_{2}}\right)}}
\end{aligned}
$$


with the help of which the matrix elements between antisymmetric states can be deduced.

\subsection{Correlations between two particles in a single shell}

With these preliminaries the correlation functions $\mathcal{C}_{(n \ell)^{2} ; L S}(r, R)$ can now be investigated for two particles in a single- $\ell$ shell. This correlation function possesses a symmetry related to the parity quantum number (see, e.g., Refs. $[15,16]$ ). Let $\hat{P}$ represent the parity operator that takes $\bar{r}$ to $-\bar{r}, \hat{P}|\bar{r}\rangle=|-\bar{r}\rangle$. This operator is hermitian, $\hat{P}^{\dagger}=\hat{P}$, with eigenvalues \pm 1 , and $\hat{P}^{2}$ is the identity operator. Parity operators for different particles are distinguished by the subscript $i$, so that $\hat{P}_{i}$ is the parity operator acting on the coordinates of particle $i$. With use of the properties

$$
\hat{P}_{1} \delta\left(r-r_{12}\right) \delta\left(R-R_{12}\right)=\delta\left(r-2 R_{12}\right) \delta\left(R-r_{12} / 2\right)
$$

and

$$
\hat{P}_{1}\left|(n \ell)^{2} ; L M_{L} S M_{S}\right\rangle=(-)^{\ell}\left|(n \ell)^{2} ; L M_{L} S M_{S}\right\rangle,
$$

the following identity can be established:

$$
\begin{aligned}
& \left\langle(n \ell)^{2} ; L M_{L} S M_{S}\left|\hat{P}_{1} \delta\left(r-r_{12}\right) \delta\left(R-R_{12}\right)\right|(n \ell)^{2} ; L M_{L} S M_{S}\right\rangle \\
& =\left\langle(n \ell)^{2} ; L M_{L} S M_{S}\right|\left(\hat{P}_{1} \delta\left(r-r_{12}\right) \delta\left(R-R_{12}\right)\left|(n \ell)^{2} ; L M_{L} S M_{S}\right\rangle\right) \\
& =(-)^{\ell}\left\langle(n \ell)^{2} ; L M_{L} S M_{S}\left|\delta\left(r / 2-R_{12}\right) \delta\left(2 R-r_{12}\right)\right|(n \ell)^{2} ; L M_{L} S M_{S}\right\rangle .
\end{aligned}
$$

On the other hand, since $\hat{P}_{i}$ is hermitian, the following identity is valid:

$$
\begin{aligned}
& \left\langle(n \ell)^{2} ; L M_{L} S M_{S}\left|\hat{P}_{1} \delta\left(r-r_{12}\right) \delta\left(R-R_{12}\right)\right|(n \ell)^{2} ; L M_{L} S M_{S}\right\rangle \\
& =\left(\left\langle(n \ell)^{2} ; L M_{L} S M_{S}\right| \hat{P}_{1}\right) \delta\left(r-r_{12}\right) \delta\left(R-R_{12}\right)\left|(n \ell)^{2} ; L M_{L} S M_{S}\right\rangle \\
& =(-1)^{\ell}\left\langle(n \ell)^{2} ; L M_{L} S M_{S}\left|\delta\left(r-r_{12}\right) \delta\left(R-R_{12}\right)\right|(n \ell)^{2} ; L M_{L} S M_{S}\right\rangle .
\end{aligned}
$$

It therefore follows that in a single- $\ell$ shell the two-particle correlation function must satisfy the property

$$
\mathcal{C}_{(n \ell)^{2} ; L S}(r, R)=\mathcal{C}_{(n \ell)^{2} ; L S}(2 R, r / 2),
$$

which corresponds to a "reflection-like" symmetry about the plane $r=2 R$ that will be present throughout this section.

Let us now illustrate the character of the two-particle correlation function $\mathcal{C}_{\alpha}(r, R)$ for a variety of states $|\alpha\rangle$. Figure 1 shows the correlation function for different $s$ shells. Since $\ell=0$ in this case, it follows necessarily that $L=0$ and 


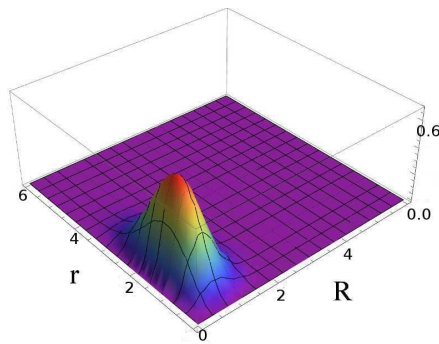

(a) $\left|(0 s)^{2} ; 00\right\rangle$

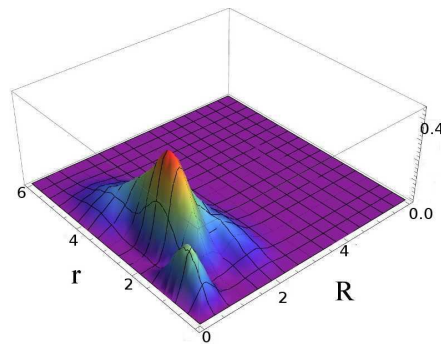

(b) $\left|(1 s)^{2} ; 00\right\rangle$

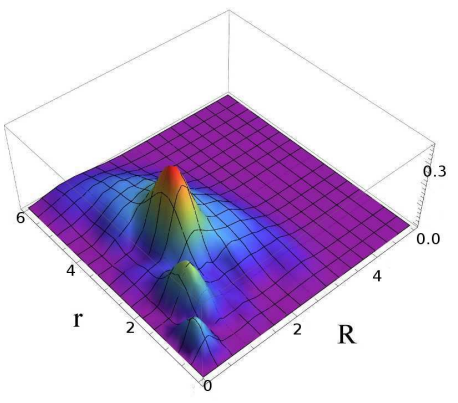

(c) $\left|(2 s)^{2} ; 00\right\rangle$

Fig. 1. The correlation function $\mathcal{C}_{\alpha}(r, R)$ for the two-particle configurations $|\alpha\rangle=\left|(n s)^{2} ; L=S=0\right\rangle$ with radial quantum number $n=0,1$ and 2 .

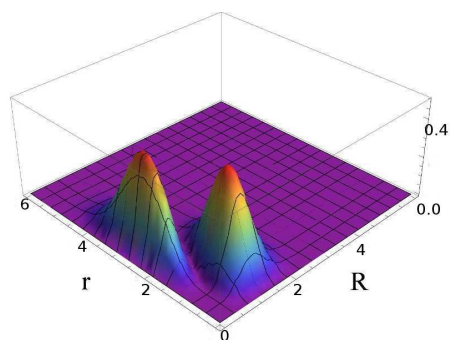

(a) $\left|(0 p)^{2} ; 00\right\rangle$

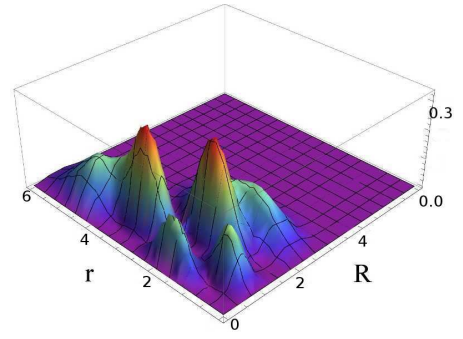

(d) $\left|(1 p)^{2} ; 00\right\rangle$

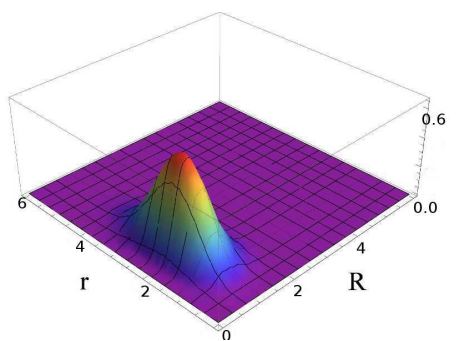

(b) $\left|(0 p)^{2} ; 11\right\rangle$

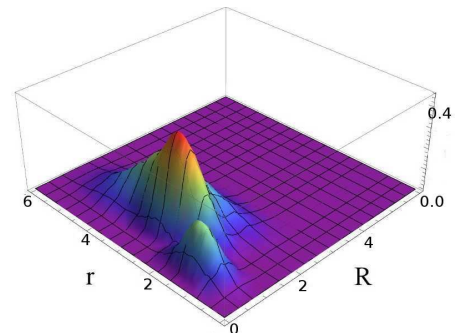

(e) $\left|(1 p)^{2} ; 11\right\rangle$

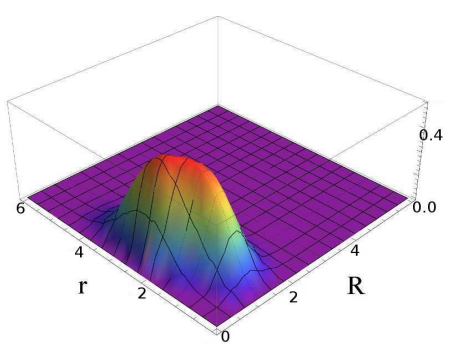

(c) $\left|(0 p)^{2} ; 20\right\rangle$

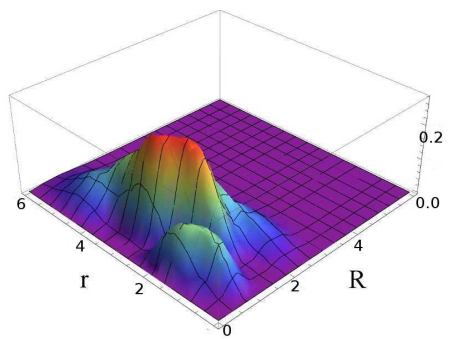

(f) $\left|(1 p)^{2} ; 20\right\rangle$

Fig. 2. The correlation function $\mathcal{C}_{\alpha}(r, R)$ for the two-particle configurations $|\alpha\rangle=\left|(n p)^{2} ; L S\right\rangle$ with total orbital angular momentum and $\operatorname{spin}(L S)=(00)$, (11) and (20), and with radial quantum number $n=0$ and 1.

$S=0$, and that the spatial wave function is symmetric. For a state $\left|(n s)^{2} ; 00\right\rangle$ with radial quantum number $n, n+1$ maxima are observed. Starting from $n=0$, where there is one maximum, the pattern repeats itself as $n$ increases with higher intensity while at the same time spanning out to larger radii. The $n+1$ maxima are positioned in $n+1$ concentric rings radiating out from the center and situated on the line $r=2 R$. As the maximum goes out to larger radii, it also becomes higher. The recurring pattern can be understood as the effect of nodes of the radial wave functions. A radial wave function characterized by the quantum number $n$ has $n$ nodes, giving rise to $n+1$ rings.

The three possible configurations for the $0 p$ shell have $(L S)=(00),(11)$ and 
(20), whose correlation functions are shown in the upper part of Fig. 2. Two maxima are observed for $\left|(0 p)^{2} ; 00\right\rangle$ and one for $\left|(0 p)^{2} ; 11\right\rangle$ whereas an indistinct coexistence of two maxima can be perceived in the $\left|(0 p)^{2} ; 11\right\rangle$ correlation.

A closer look at the form of correlation function of the three possible states in the $0 p$ shell may shed some light on these results. The correlation functions for the three configurations are

$$
\begin{aligned}
& \mathcal{C}_{(0 p)^{2} ; 00}(r, R)=\frac{4}{3 \pi}\left(r^{6} R^{2}-8 r^{4} R^{4}+16 r^{2} R^{6}\right) e^{-r^{2} / 2-2 R^{2}} \\
& \mathcal{C}_{(0 p)^{2} ; 11}(r, R)=\frac{64}{9 \pi} r^{4} R^{4} e^{-r^{2} / 2-2 R^{2}} \\
& \mathcal{C}_{(0 p)^{2} ; 20}(r, R)=\frac{8}{15 \pi}\left(r^{6} R^{2}+16 r^{2} R^{6}\right) e^{-r^{2} / 2-2 R^{2}}
\end{aligned}
$$

Consistent with the relation (14), the correlation function $\mathcal{C}_{(0 p)^{2} ; 00}(r, R)$ has two maxima at

$$
(r, R)=\left(\sqrt{2(2 \pm \sqrt{2})}, \sqrt{\frac{1}{2}(2 \mp \sqrt{2})}\right)
$$

and $\mathcal{C}_{(0 p)^{2} ; 11}(r, R)$ has only one maximum at $(r, R)=(2,1)$. As for $\mathcal{C}_{(0 p)^{2} ; 20}(r, R)$, it is the linear combination

$$
\mathcal{C}_{(0 p)^{2} ; 20}(r, R)=\frac{2}{5} \mathcal{C}_{(0 p)^{2} ; 00}(r, R)+\frac{3}{5} \mathcal{C}_{(0 p)^{2} ; 11}(r, R),
$$

which explains the vaguely perceivable two-maximum structure.

By analogy with the correlation function for the $n s$ shells, one expects that each plot for the $0 p$ shell is repeated for the $1 p$ shell, in a ring farther away from the origin with higher intensity. This is confirmed by the examples shown in the lower part of Fig. 2.

In addition to the pattern concerning the radial quantum number $n$, as one accesses higher shells, another pattern related to $\ell$ becomes apparent. As illustrated by the correlation function for configurations in the $0 d$ shell in Fig. 3, if $\ell$ increases by one from $p$ to $d$, one more maximum is found for the state with $(L S)=(00)$. Another pattern worth mentioning is that for the lowest- $L$ configurations, the number of maxima decreases by one as the total orbital angular momentum $L$ increases by one.

The patterns described above are confirmed as one examines other shells. For of shell, as shown in Fig. 4, the $(L S)=(00)$, (11) and (20) configurations exhibit four, three and two maxima, respectively. For higher total orbital angular momenta the plots are less distinctly defined. 


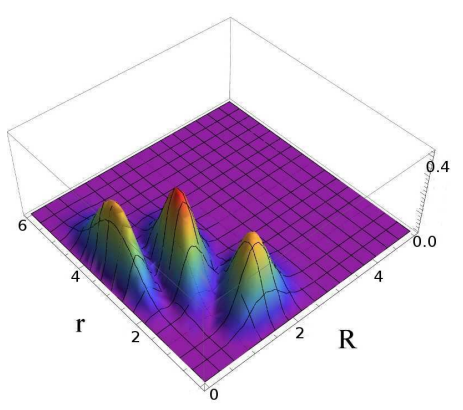

(a) $\left|(0 d)^{2} ; 00\right\rangle$

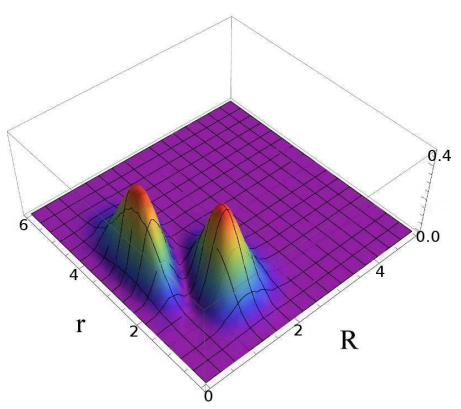

(b) $\left|(0 d)^{2} ; 11\right\rangle$

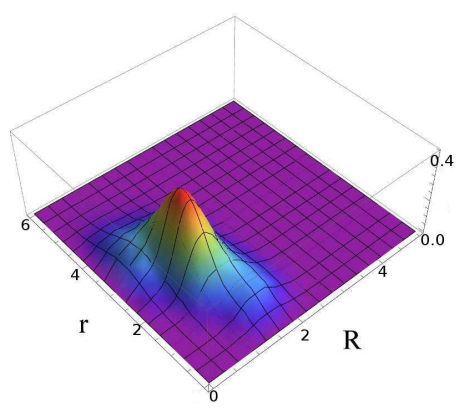

(c) $\left|(0 d)^{2} ; 20\right\rangle$

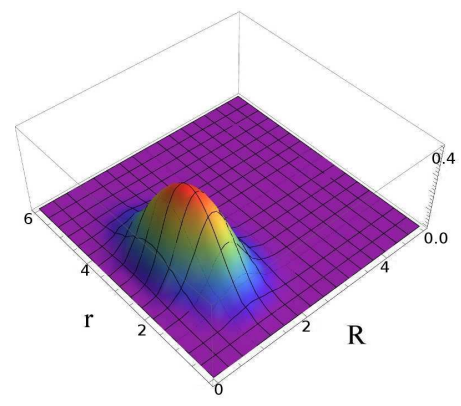

(d) $\left|(0 d)^{2} ; 31\right\rangle$

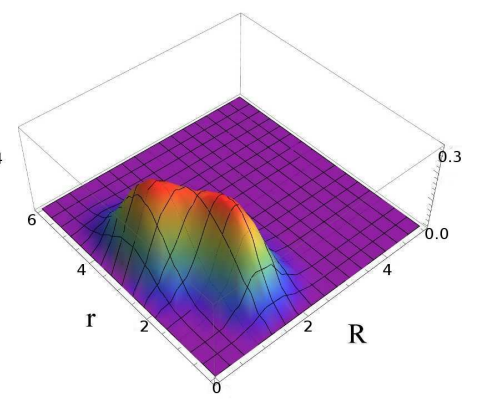

(e) $\left|(0 d)^{2} ; 40\right\rangle$

Fig. 3. The correlation function $\mathcal{C}_{\alpha}(r, R)$ for the two-particle configurations $|\alpha\rangle=\left|(0 d)^{2} ; L S\right\rangle$ with total orbital angular momentum and spin $(L S)=(00)$, (11), (20), (31) and (40).

To summarize, other than the reflection-like symmetry (14), the correlation function of two particles in the $n \ell$ shell coupled to total angular momentum $L$ exhibits a few patterns related to $n, \ell$ and $L$. Such a correlation function displays an $(n+1)$-circle structure in that, compared to the maxima observed for the configuration $\left|(0 \ell)^{2} ; L S\right\rangle, n+1$ times as many peaks positioned on $n+1$ concentric circles radiating out from the center exist for $\left|(n \ell)^{2} ; L S\right\rangle$. This is so because the radial wave function $R_{n \ell}(r)$ has $n$ nodes and hence its square has $n+1$ maxima. On each circle, the number of maxima is related to $\ell$ and $L$. For $L=0$, there are $\ell+1$ maxima on each circle. As $L$ increases by one, the number of maxima decreases by one for the first few $L$. For large values of $L$, the peak shapes become less defined. Catara et al. [15], who studied two-particle correlations in the $j j$-coupling scheme, made similar but less conclusive observations, and did not give the analytical explanation of the observed regularities which is given here in Subsect. 3.5.

\subsection{Correlations between two particles in different shells}

In this subsection the properties are studied of correlation functions of the type $\mathcal{C}_{\alpha \alpha^{\prime}}(r, R)$, where $|\alpha\rangle$ and $\left|\alpha^{\prime}\right\rangle$ stand for two-particle states in different shells, $\left|(n \ell)^{2} ; L S\right\rangle$ and $\left|\left(n^{\prime} \ell^{\prime}\right)^{2} ; L S\right\rangle$, respectively. As opposed to $\mathcal{C}_{\alpha}(r, R)$, which 


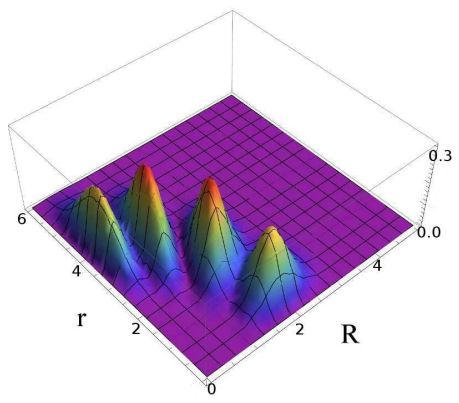

(a) $\left|(0 f)^{2} ; 00\right\rangle$

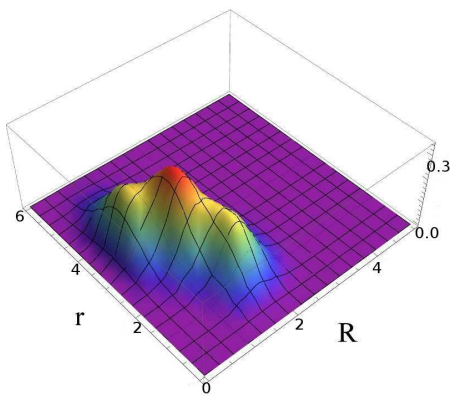

(d) $\left|(0 f)^{2} ; 31\right\rangle$

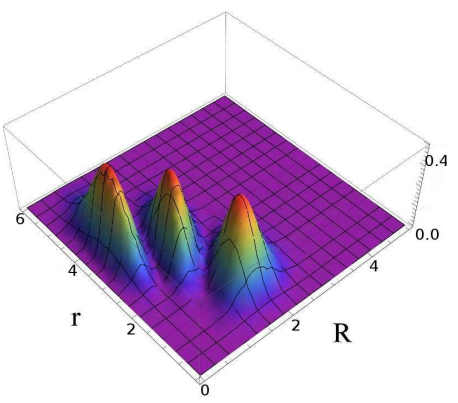

(b) $\left|(0 f)^{2} ; 11\right\rangle$

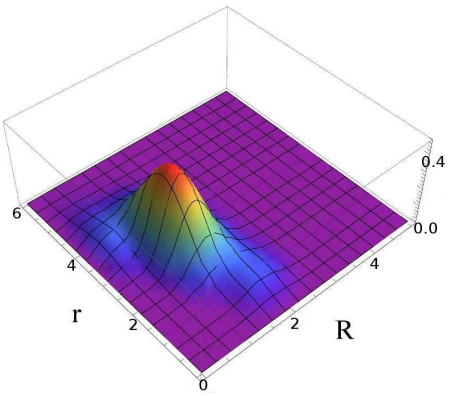

(e) $\left|(0 f)^{2} ; 40\right\rangle$

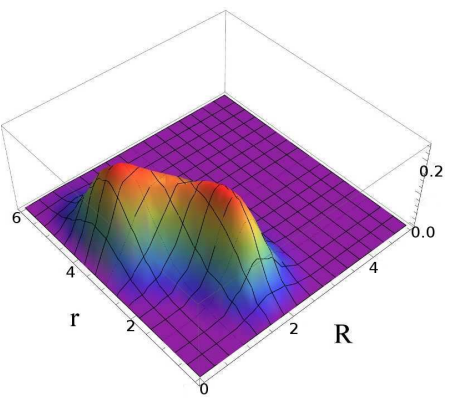

(g) $\left|(0 f)^{2} ; 60\right\rangle$

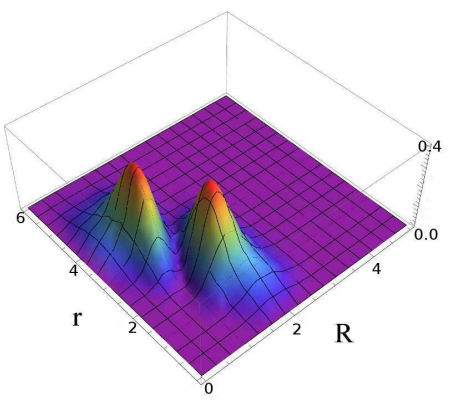

(c) $\left|(0 f)^{2} ; 20\right\rangle$

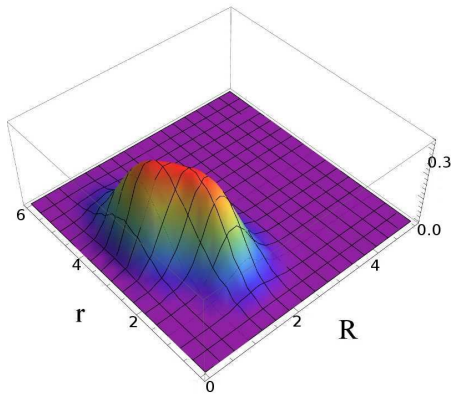

(f) $\left|(0 f)^{2} ; 51\right\rangle$

Fig. 4. The correlation function $\mathcal{C}_{\alpha}(r, R)$ for the two-particle configurations $|\alpha\rangle=\left|(0 f)^{2} ; L S\right\rangle$ with total orbital angular momentum and spin $(L S)=(00)$, (11), (20), (31), (40), (51) and (60).

has its physical interpretation as the probability distribution of the state $|\alpha\rangle$ as a function of $r$ and $R, \mathcal{C}_{\alpha \alpha^{\prime}}(r, R)$ makes for better understanding of the interference of contributions from different shells. Such an understanding is useful when studying the probability distribution of any two-particle state that is an admixture of different shells.

To examine the role of parity, examples of correlation functions $\mathcal{C}_{\alpha \alpha^{\prime}}(r, R)$ between two shells of the same parity and between two shells of opposite parity are presented in Fig. 5 and Fig. 6, respectively. By way of an argument similar to the one leading to Eq. (14), the following property can be shown to be valid:

$$
(-)^{\ell} \mathcal{C}_{(n \ell)^{2} ; L S,\left(n^{\prime} \ell^{\prime}\right)^{2} ; L S}(r, R)=(-)^{\ell^{\prime}} \mathcal{C}_{(n \ell)^{2} ; L S,\left(n^{\prime} \ell^{\prime}\right)^{2} ; L S}(2 R, r / 2)
$$




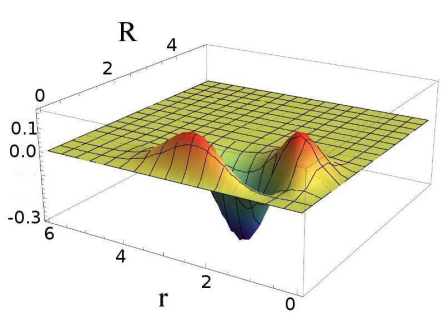

(a) $\left|(0 s)^{2} ; 00\right\rangle,\left|(0 d)^{2} ; 00\right\rangle$

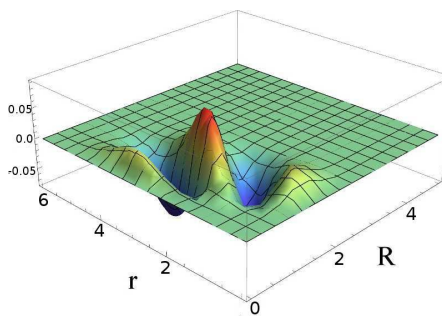

(b) $\left|(0 s)^{2} ; 00\right\rangle,\left|(0 g)^{2} ; 00\right\rangle$

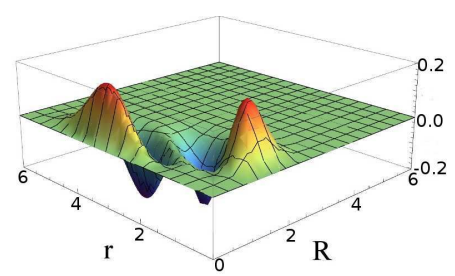

(c) $\left|(0 p)^{2} ; 00\right\rangle,\left|(0 f)^{2} ; 00\right\rangle$

Fig. 5. The correlation function $\mathcal{C}_{\alpha \alpha^{\prime}}(r, R)$ for two-particle configurations $|\alpha\rangle \neq\left|\alpha^{\prime}\right\rangle$ with the same parity.

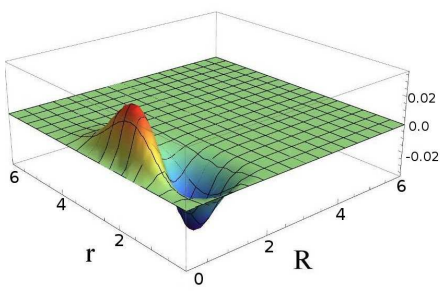

(a) $\left|(0 s)^{2} ; 00\right\rangle,\left|(0 p)^{2} ; 00\right\rangle$

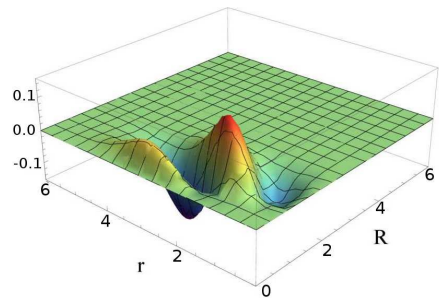

(b) $\left|(0 s)^{2} ; 00\right\rangle,\left|(0 f)^{2} ; 00\right\rangle$

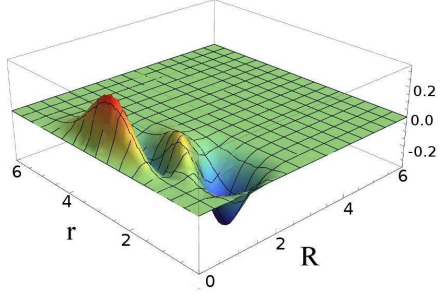

(c) $\left|(0 p)^{2} ; 00\right\rangle,\left|(0 d)^{2} ; 00\right\rangle$

Fig. 6. The correlation function $\mathcal{C}_{\alpha \alpha^{\prime}}(r, R)$ for two-particle configurations $|\alpha\rangle \neq\left|\alpha^{\prime}\right\rangle$ with different parity.

Therefore, between a pair of shells of the same parity, $\mathcal{C}_{\alpha \alpha^{\prime}}(r, R)$ has a reflectionlike symmetry as $\mathcal{C}_{\alpha}(r, R)$ does, obeying the relation (14). Between a pair of shells of opposite parity, the appropriate relation becomes

$$
\mathcal{C}_{\alpha \alpha^{\prime}}(r, R)=-\mathcal{C}_{\alpha \alpha^{\prime}}(2 R, r / 2)
$$

giving rise to a "rotation-like" symmetry with respect to the line $r=2 R$.

\subsection{The angular correlation function}

Spatial correlations between two particles should also be reflected in their angular separation, that is, the relative angle $\theta_{12}$ between them. While the results of the previous subsection are obtained with the functions (1) in terms of the relative distance $r_{12}$ between the particles and the distance $R_{12}$ of their center of mass from the origin, they can also be studied from a different angle by deriving, for a given two-particle state, the probability density as a function of $\theta_{12}$.

To illustrate the essential idea of the method, consider a state of two particles in a single- $\ell$ shell coupled to orbital angular momentum $L=0$. Its wave function can be expressed as 


$$
\begin{aligned}
& \left\langle\bar{r}_{1}, \bar{r}_{2} \mid n_{1} \ell, n_{2} \ell ; L=M_{L}=0\right\rangle \\
& =R_{n_{1} \ell}\left(r_{1}\right) R_{n_{2} \ell}\left(r_{2}\right)\left[Y_{\ell}\left(\Omega_{1}\right) \times Y_{\ell}\left(\Omega_{2}\right)\right]_{0}^{(0)} \\
& =R_{n_{1} \ell}\left(r_{1}\right) R_{n_{2} \ell}\left(r_{2}\right) \sum_{m=-\ell}^{\ell} \frac{(-)^{\ell-m}}{\sqrt{2 \ell+1}}(-)^{m} Y_{\ell m}\left(\Omega_{1}\right) Y_{\ell m}^{*}\left(\Omega_{2}\right) \\
& =R_{n_{1} \ell}\left(r_{1}\right) R_{n_{2} \ell}\left(r_{2}\right)(-)^{\ell} \frac{\sqrt{2 \ell+1}}{4 \pi} P_{\ell}\left(\cos \theta_{12}\right) .
\end{aligned}
$$

In the last step use is made of the addition theorem for spherical harmonics [13], with $P_{\ell}(x)$ a Legendre polynomial. The angular part of the wave function, therefore, is a function of the relative angle $\theta_{12}$ between the two particles.

This result can be generalized to the case of a two-particle state in two different shells coupled to any total orbital angular momentum $L$ and total spin $S$, $\left|n_{1} \ell_{1}, n_{2} \ell_{2} ; L M_{L} S M_{S}\right\rangle$. To obtain its probability density, the average over all projections $M_{L}$ and $M_{S}$ should be taken. Since the angular part is independent of $M_{S}$, the following quantity must be evaluated:

$$
\frac{1}{2 L+1} \sum_{M_{L}}\left[Y_{\ell_{1}}^{*}\left(\Omega_{1}\right) \times Y_{\ell_{2}}^{*}\left(\Omega_{2}\right)\right]_{M_{L}}^{(L)}\left[Y_{\ell_{1}}\left(\Omega_{1}\right) \times Y_{\ell_{2}}\left(\Omega_{2}\right)\right]_{M_{L}}^{(L)}
$$

A closed expression can be obtained by use of the general result

$$
\begin{aligned}
& \frac{1}{2 L+1} \sum_{M_{L}}\left[Y_{\ell_{1}}^{*}\left(\Omega_{1}\right) \times Y_{\ell_{2}}^{*}\left(\Omega_{2}\right)\right]_{M_{L}}^{(L)}\left[Y_{\ell_{1}^{\prime}}\left(\Omega_{1}\right) \times Y_{\ell_{2}^{\prime}}\left(\Omega_{2}\right)\right]_{M_{L}}^{(L)} \\
= & \frac{1}{16 \pi^{2}} \sqrt{\left(2 \ell_{1}+1\right)\left(2 \ell_{2}+1\right)\left(2 \ell_{1}^{\prime}+1\right)\left(2 \ell_{2}^{\prime}+1\right)}(-)^{L} \sum_{\lambda}(-)^{\lambda}(2 \lambda+1) \\
& \times\left\{\begin{array}{lll}
\ell_{2} & \ell_{2}^{\prime} & \lambda \\
\ell_{1}^{\prime} & \ell_{1} & L
\end{array}\right\}\left(\begin{array}{ccc}
\ell_{1} & \ell_{1}^{\prime} & \lambda \\
0 & 0 & 0
\end{array}\right)\left(\begin{array}{ccc}
\ell_{2} & \ell_{2}^{\prime} & \lambda \\
0 & 0 & 0
\end{array}\right) P_{\lambda}\left(\cos \theta_{12}\right) .
\end{aligned}
$$

This is a Legendre-polynomial expansion having the cosine of the relative angle $\theta_{12}$ as its argument; $\ell_{1}+\ell_{1}^{\prime}$ and $\ell_{2}+\ell_{2}^{\prime}$ have to be of the same parity, otherwise the expression (22) vanishes. More specifically, if $\ell_{1}+\ell_{1}^{\prime}$ and $\ell_{2}+\ell_{2}^{\prime}$ are even, it is an expansion in even-degree Legendre polynomials whereas if $\ell_{1}+\ell_{1}^{\prime}$ and $\ell_{2}+\ell_{2}^{\prime}$ are odd, it is an expansion in odd-degree Legendre polynomials.

Availing of the result (22) involving two two-particle states $\left|\ell_{1} \ell_{2} ; L M_{L} S M_{S}\right\rangle$ and $\left|\ell_{1}^{\prime} \ell_{2}^{\prime} ; L M_{L} S M_{S}\right\rangle$, we can evaluate the angular probability density of two particles in the same $n \ell$ shell or in two shells characterized by the same $\ell$, coupled to total orbital angular momentum $L$ and total spin $S$. It takes the form of an expansion of even-degree Legendre polynomials, ${ }^{1}$

$\overline{1}$ There is no dependence on the radial quantum number $n$ which is therefore omit- 


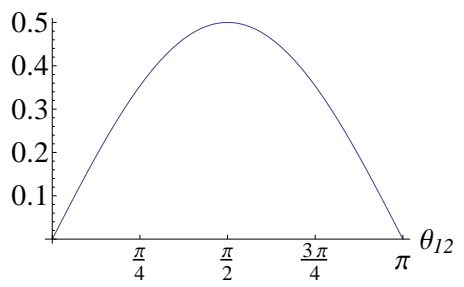

Fig. 7. The angular correlation function $\tilde{\mathcal{C}}_{\alpha}\left(\theta_{12}\right)$ for the two-particle configuration $|\alpha\rangle=\left|s^{2} ; L=S=0\right\rangle$.

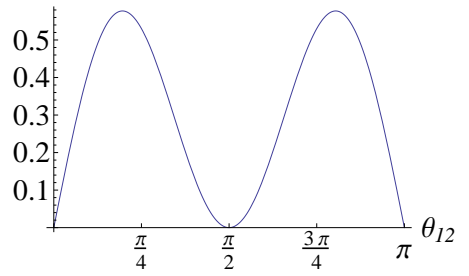

(a) $\left|p^{2} ; 00\right\rangle$

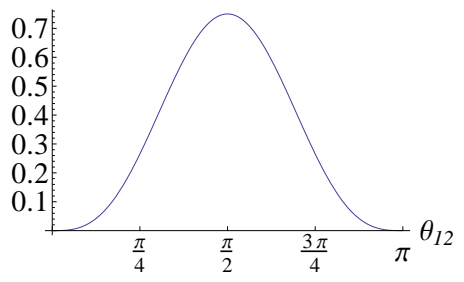

(b) $\left|p^{2} ; 11\right\rangle$

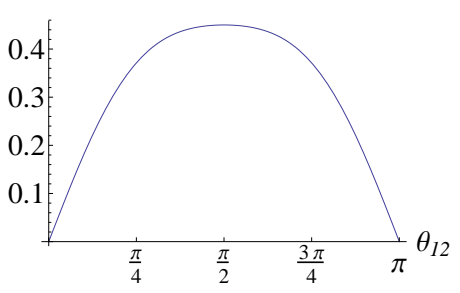

(c) $\left|p^{2} ; 20\right\rangle$

Fig. 8. The angular correlation function $\tilde{\mathcal{C}}_{\alpha}\left(\theta_{12}\right)$ for the two-particle configurations $|\alpha\rangle=\left|p^{2} ; L S\right\rangle$ with $(L S)=(00),(11)$ and $(20)$.

$$
\begin{aligned}
\mathcal{P}_{\ell^{2} ; L S}\left(\theta_{12}\right) & \equiv \frac{1}{2 L+1} \sum_{M_{L}}\left[Y_{\ell}^{*}\left(\Omega_{1}\right) \times Y_{\ell}^{*}\left(\Omega_{2}\right)\right]_{M_{L}}^{(L)}\left[Y_{\ell}\left(\Omega_{1}\right) \times Y_{\ell}\left(\Omega_{2}\right)\right]_{M_{L}}^{(L)} \\
& =\frac{(2 \ell+1)^{2}}{16 \pi^{2}}(-)^{L} \sum_{\lambda}(2 \lambda+1)\left\{\begin{array}{lll}
\ell & \ell & \lambda \\
\ell & \ell & L
\end{array}\right\}\left(\begin{array}{lll}
\ell & \ell & \lambda \\
0 & 0 & 0
\end{array}\right)^{2} P_{\lambda}\left(\cos \theta_{12}\right) .
\end{aligned}
$$

Assume that one of the particles is in the zenith direction and hence the angular separation $\theta_{12}$ is the polar angle. The probability of finding the other particle in the differential solid angle element $d \Omega$ is $4 \pi \mathcal{P}_{\ell^{2} ; L S}\left(\theta_{12}\right) \sin \theta_{12} d \theta_{12} d \phi$. Integration over $\phi$ yields the expression for the probability of finding the other particle within an angle $d \theta_{12}$. The corresponding probability density

$$
\tilde{\mathcal{C}}_{\ell^{2} ; L S}\left(\theta_{12}\right)=8 \pi^{2} \mathcal{P}_{\ell^{2} ; L S}\left(\theta_{12}\right) \sin \theta_{12},
$$

shall be referred to as the angular correlation function.

In Figs. 7 to 10 are plotted the angular correlation functions for the $s, p, d$ and $f$ shells. They are to be compared with Figs. 1 to 4 . Both sets are illustrations, but from different perspectives, of the spatial structure of a pair of particles in a single- $\ell$ shell. Figures 7 to 10 can be best described as the projected 2D images along the direction $r=2 R$ of the Figs. 1 to 4 and reinforce the observations made in Subsect. 3.3 about the patterns related to $\ell$ and $L$. In fact, a study of the properties of the angular correlation function for two particles coupled to total orbital angular momentum $L=0$ and total spin

ted henceforth in this subsection. 


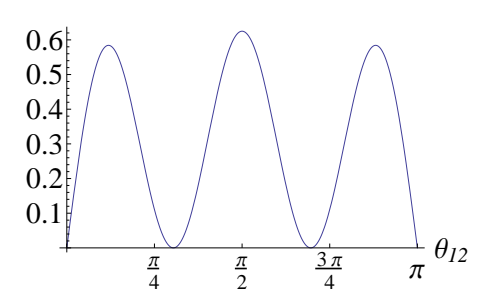

(a) $\left|d^{2} ; 00\right\rangle$

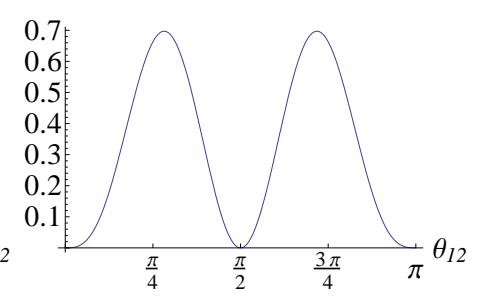

(b) $\left|d^{2} ; 11\right\rangle$

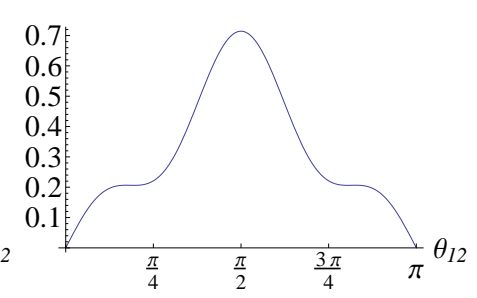

(c) $\left|d^{2} ; 20\right\rangle$

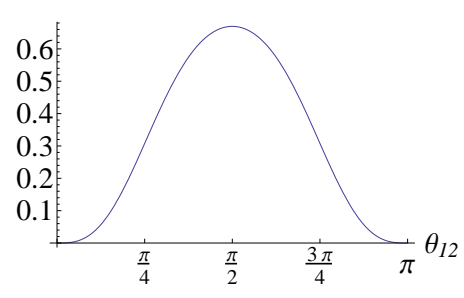

(d) $\left|d^{2} ; 31\right\rangle$

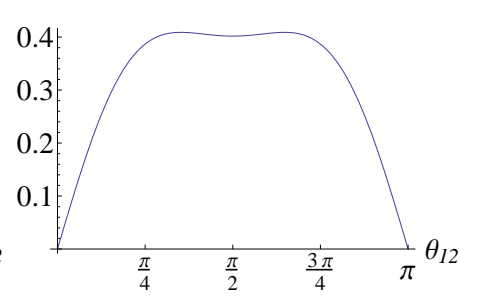

(e) $\left|d^{2} ; 40\right\rangle$

Fig. 9. The angular correlation function $\tilde{\mathcal{C}}_{\alpha}\left(\theta_{12}\right)$ for the two-particle configurations $|\alpha\rangle=\left|d^{2} ; L S\right\rangle$ with $(L S)=(00),(11),(20),(31)$ and (40).

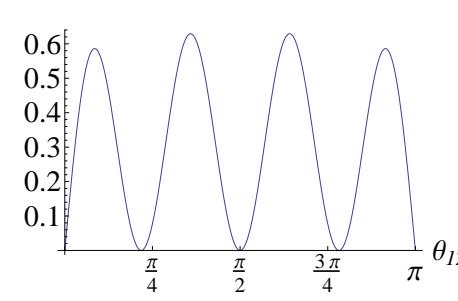

(a) $\left|f^{2} ; 00\right\rangle$

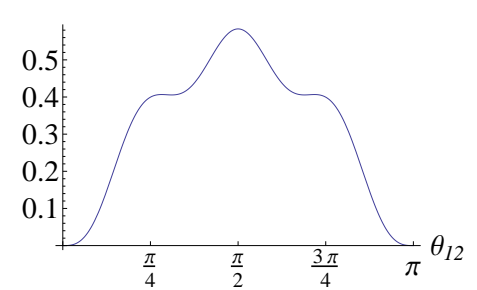

(d) $\left|f^{2} ; 31\right\rangle$

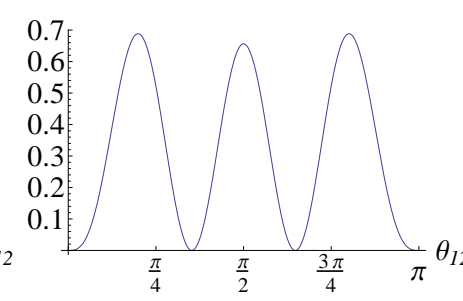

(b) $\left|f^{2} ; 11\right\rangle$

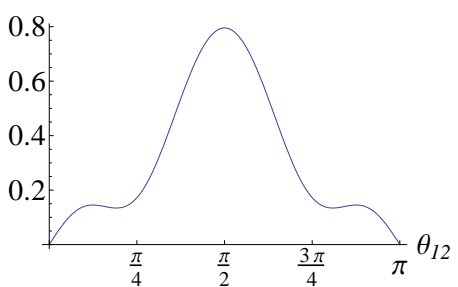

(e) $\left|f^{2} ; 40\right\rangle$

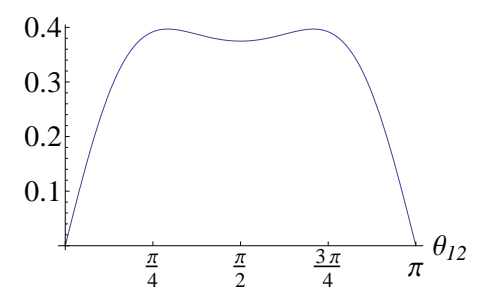

(g) $\left|f^{2} ; 60\right\rangle$

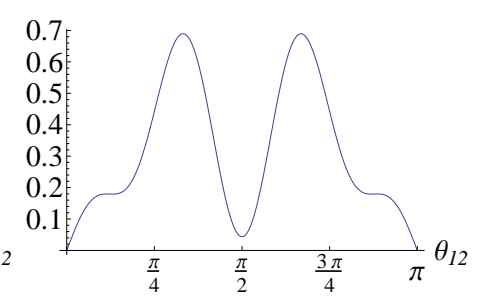

(c) $\left|f^{2} ; 20\right\rangle$

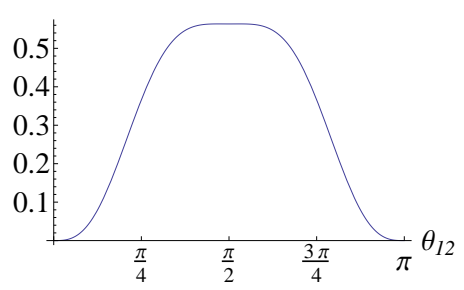

(f) $\left|f^{2} ; 51\right\rangle$

Fig. 10. The angular correlation function $\tilde{\mathcal{C}}_{\alpha}\left(\theta_{12}\right)$ for the two-particle configurations $|\alpha\rangle=\left|f^{2} ; L S\right\rangle$ with $(L S)=(00),(11),(20),(31),(40),(51)$ and (60). 
$S=0$ shows that $\tilde{\mathcal{C}}_{\ell^{2} ; 00}\left(\theta_{12}\right)$ has $\ell+1$ distinct maxima. This is because the angular correlation function in this case

$$
\tilde{\mathcal{C}}_{\ell^{2} ; 00}\left(\theta_{12}\right)=\frac{2 \ell+1}{2} P_{\ell}^{2}\left(\cos \theta_{12}\right) \sin \theta_{12}
$$

is proportional to the square of the $\ell$-th degree Legendre polynomial. Furthermore, it can also be shown with the help of the expression (23) that this number of maxima decreases by one if the total orbital angular momentum increases to $L=1$, as the angular correlation function

$$
\tilde{\mathcal{C}}_{\ell^{2} ; 11}\left(\theta_{12}\right)=\frac{2 \ell+1}{2 \ell(\ell+1)} \sin ^{3} \theta_{12} P_{\ell}^{\prime 2}\left(\cos \theta_{12}\right)
$$

is proportional to the square of the derivative of the $\ell$-th degree Legendre polynomial. For higher $L$ values the maxima of the angular correlation function $\tilde{\mathcal{C}}_{\ell^{2} ; L S}\left(\theta_{12}\right)$ become less distinct which also coincides with the conclusion of Subsect. 3.3.

\subsection{Angular correlations between two particles in different shells}

In the same manner the angular correlations can be studied between two particles in different shells $\ell$ and $\ell^{\prime}$. The relevant expression for the angular correlation function is

$$
\begin{aligned}
\tilde{\mathcal{C}}_{\ell \ell^{\prime} ; L S}\left(\theta_{12}\right)= & \frac{1}{2}(2 \ell+1)\left(2 \ell^{\prime}+1\right)(-)^{L} \\
& \times \sum_{\lambda}(-)^{\lambda}(2 \lambda+1)\left\{\begin{array}{lll}
\ell & \ell^{\prime} & \lambda \\
\ell^{\prime} & \ell & L
\end{array}\right\}\left(\begin{array}{lll}
\ell & \ell^{\prime} & \lambda \\
0 & 0 & 0
\end{array}\right)^{2} P_{\lambda}\left(\cos \theta_{12}\right) \sin \theta_{12} .
\end{aligned}
$$

Some examples are shown in Fig. 11. They are projected 2D images of the $3 \mathrm{D}$ plots of correlation function in Figs. 5 and 6 . The reflection-like symmetry about the line $\theta_{12}=\frac{1}{2} \pi$ for angular correlation functions between shells of the same parity and the rotational-like symmetry around the point $\left(\frac{1}{2} \pi, 0\right)$ for those between shells of opposite parity are determined by the Wigner $3 j$ symbol in the expression (27). If $\ell$ and $\ell^{\prime}$ are both even or both odd, $\lambda$ must be even, resulting in symmetric Legendre polynomials $P_{\lambda}(x)$ and a reflection-symmetric angular correlation function $\tilde{\mathcal{C}}_{\ell \ell^{\prime} ; L S}\left(\theta_{12}\right)$; otherwise, only anti-symmetric Legendre polynomials contribute and the rotational symmetry ensues. 


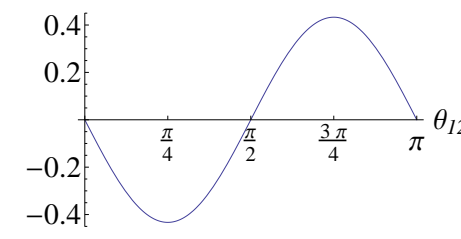

(a) $\left|s^{2} ; 00\right\rangle,\left|p^{2} ; 00\right\rangle$

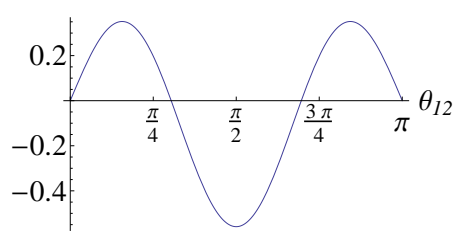

(b) $\left|s^{2} ; 00\right\rangle,\left|d^{2} ; 00\right\rangle$

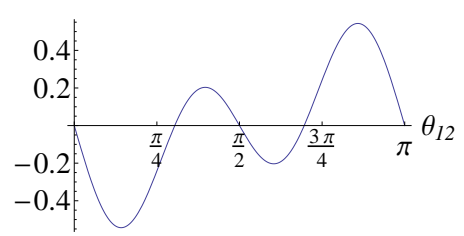

(c) $\left|p^{2} ; 00\right\rangle,\left|d^{2} ; 00\right\rangle$

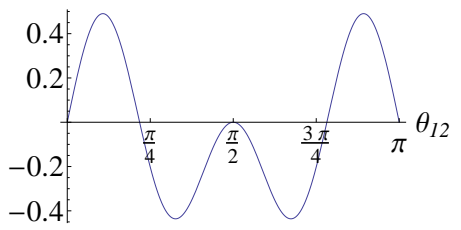

(d) $\left|p^{2} ; 00\right\rangle,\left|f^{2} ; 00\right\rangle$

Fig. 11. The angular correlation function $\tilde{\mathcal{C}}_{\alpha \alpha^{\prime}}\left(\theta_{12}\right)$ for two-particle configurations $|\alpha\rangle \neq\left|\alpha^{\prime}\right\rangle$.

\section{Two-particle correlations in ${ }^{6} \mathrm{He}$}

Let us now apply to the nucleus ${ }^{6} \mathrm{He}$ what has been formulated in Sect. 3 . It is assumed that the $\alpha$ particle is inert, the $0 s$ shell being occupied by two neutrons and two protons, so that only the two valence neutrons come into play. A residual interaction should be considered between the two valence neutrons which is taken to be a schematic delta interaction. The valence neutrons are first assumed to occupy the $0 p$ shell and, subsequently, scattering into higher shells is allowed for. In the latter case, spurious center-of-mass motion must be eliminated which can be achieved in the framework of the cluster-orbital shell model. In a final subsection, phenomenological estimates are derived for the oscillator length and the interaction strength, to arrive at a quantitative prediction of the two-particle correlations in ${ }^{6} \mathrm{He}$.

\subsection{The delta interaction}

The matrix elements of the delta function $\delta\left(\bar{r}_{1}-\bar{r}_{2}\right)$ are obtained by putting $\bar{r}=\overline{0}$ in the expression (6). Since only $s$ waves do not vanish at the origin, one must have $\ell=\ell^{\prime}=0$ which in turn implies $\lambda=\mu=0, L=L^{\prime}=\mathcal{L}$ and $M_{L}=M_{L}^{\prime}$. The expression (6) then reduces to

$$
\begin{aligned}
& \left\langle n_{1} \ell_{1} n_{2} \ell_{2} ; L M_{L} S M_{S}\left|\delta\left(\bar{r}_{1}-\bar{r}_{2}\right)\right| n_{1}^{\prime} \ell_{1}^{\prime} n_{2}^{\prime} \ell_{2}^{\prime} ; L^{\prime} M_{L}^{\prime} S^{\prime} M_{S}^{\prime}\right\rangle \\
& =\delta_{L L^{\prime}} \delta_{M_{L} M_{L}^{\prime}} \delta_{S S^{\prime}} \delta_{M_{S} M_{S}^{\prime}} \frac{1}{8 \pi \sqrt{2}} \sum_{n n^{\prime} \mathcal{N}} a_{n 0 \mathcal{N} L, L}^{n_{1} \ell_{1} n_{2} \ell_{2}} a_{n^{\prime} 0 \mathcal{N} L, L}^{n_{1}^{\prime} \ell_{1}^{\prime} n_{2}^{\prime} \ell_{2}^{\prime}} R_{n 0}(0) R_{n^{\prime} 0}(0) .
\end{aligned}
$$


An alternative expression is found from the expansion

$$
\delta\left(\bar{r}_{1}-\bar{r}_{2}\right)=\frac{1}{r_{1} r_{2}} \delta\left(r_{1}-r_{2}\right) \delta\left(\cos \theta_{1}-\cos \theta_{2}\right) \delta\left(\phi_{1}-\phi_{2}\right)
$$

which, after straightforward integration and use of the properties of the spherical harmonics, leads to

$$
\begin{aligned}
& \left\langle n_{1} \ell_{1} n_{2} \ell_{2} ; L M_{L} S M_{S}\left|\delta\left(\bar{r}_{1}-\bar{r}_{2}\right)\right| n_{1}^{\prime} \ell_{1}^{\prime} n_{2}^{\prime} \ell_{2}^{\prime} ; L^{\prime} M_{L}^{\prime} S^{\prime} M_{S}^{\prime}\right\rangle \\
& =\delta_{L L^{\prime}} \delta_{M_{L} M_{L}^{\prime}} \delta_{S S^{\prime}} \delta_{M_{S}} M_{S}^{\prime} \frac{1}{4 \pi} \sqrt{\left(2 \ell_{1}+1\right)\left(2 \ell_{2}+1\right)\left(2 \ell_{1}^{\prime}+1\right)\left(2 \ell_{2}^{\prime}+1\right)} \\
& \quad \times\left(\begin{array}{ccc}
\ell_{1} & \ell_{2} & L \\
0 & 0 & 0
\end{array}\right)\left(\begin{array}{ccc}
\ell_{1}^{\prime} & \ell_{2}^{\prime} & L \\
0 & 0 & 0
\end{array}\right) \int_{0}^{+\infty} R_{n_{1} \ell_{1}}(r) R_{n_{2} \ell_{2}}(r) R_{n_{1}^{\prime} \ell_{1}^{\prime}}(r) R_{n_{2}^{\prime} \ell_{2}^{\prime}}(r) r^{2} d r .
\end{aligned}
$$

Because of the definition of the radial wave functions $R_{n \ell}(r)$ (see, e.g., Ref. [13]), the matrix elements (28) and (30) are equal to a dimensionless factor multiplied by $b^{-3}$, where $b$ is the oscillator length. The constant $g$ in the delta interaction $-g \delta\left(\bar{r}_{1}-\bar{r}_{2}\right)$, therefore, has units of $\mathrm{MeV} \mathrm{fm}{ }^{3}$.

\subsection{Two neutrons in the $0 p$ shell}

The simplest possible approach to describe the structure of the $J^{\pi}=0^{+}$ ground state of ${ }^{6} \mathrm{He}$ is to assume an inert $\alpha$-particle core with the neutron pair in the $0 p$ shell of a harmonic oscillator. If the residual interaction between the two valence neutrons is taken to be a delta interaction, then, in the limit of no spin-orbit splitting between $0 p_{3 / 2}$ and $0 p_{1 / 2}$, the Hamiltonian reads

$$
\hat{H}=\sum_{i=1}^{2}\left(\frac{1}{2 m} p_{i}^{2}+\frac{1}{2} m \omega^{2} r_{i}^{2}\right)-g \delta\left(\bar{r}_{1}-\bar{r}_{2}\right) .
$$

The $L S$ classification is exact, the ground state of ${ }^{6} \mathrm{He}$ has $L=S=0$ and the results of Sect. 3 are recovered. In particular, the correlation function shown in Fig. 2(a) applies to the ground state of the Hamiltonian (31). As argued in Sect. 3, it displays two peaks, one on each side of the line $r=2 R$, which can be identified with the di-neutron and cigar-like configurations that have been extensively discussed for ${ }^{6} \mathrm{He}[4]$.

The assumptions of no spin-orbit splitting between the $0 p_{3 / 2}$ and $0 p_{1 / 2}$ shells, of a schematic residual interaction of the delta type and of an isolated $0 p$ shell, obviously, are rather drastic approximations. Departures from the former two of these approximations can be conveniently studied by using a realistic effective interaction defined in the $0 p$ shell. In fact, a calculation confined to the $0 p$ shell with spin-orbit splitting and matrix elements as obtained by Cohen 
and Kurath [17], yields a ${ }^{6} \mathrm{He}$ ground state which is $99 \% L=S=0$ and only $1 \% L=S=1$. Therefore, essentially the same two-particle correlations are obtained in this realistic shell-model calculation as compared with those found with the schematic Hamiltonian (31). In any case, for whatever residual interaction and for arbitrary spin-orbit splitting, the symmetry property (14) requires the existence of a di-neutron and a cigar-like configuration with equal probability as long as the two valence neutrons are confined to the $0 p$ shell. Any departure from this equiprobable geometry must be the consequence of excitations of particles into other shells, which is the topic of the next subsection.

\subsection{Schematic calculations for two neutrons in many shells}

In this subsection the approximation of confining the two outer neutrons in ${ }^{6} \mathrm{He}$ to the $0 p$ shell is lifted and the effect of configuration mixing on two-particle correlations in the $0_{\mathrm{gs}}^{+}$ground state is examined. More explicitly, the changes in the correlation function $\mathcal{C}_{0_{\mathrm{ss}}^{+}}(r, R)$ are studied as a result of configuration mixing between the $0 p$ and other shells induced by the delta interaction.

If the spin-orbit interaction is neglected, the ground-state wave function and hence its two-particle correlation function, depend on a single parameter $\xi \equiv$ $g b^{-3} / \hbar \omega$, the ratio of the interaction strength $g$ times $b^{-3}$ to the spacing between two consecutive harmonic-oscillator levels, $\hbar \omega$. To gain insight into the effect of configuration mixing, first a series of schematic calculations is performed with varying $\xi$. These calculations depend on the choice of valence space, that is, the shells of the harmonic oscillator into which the two neutrons are allowed to scatter. A certain truncated valence space is chosen, specified by the number of included major shells, and the evolution of the spatial structure of the ground state is examined as a function of $\xi$.

The resulting correlation functions in the valence space comprising the shells with principal quantum numbers $1 \leq N \leq 3$ (i.e., $0 p, 1 s, 0 d, 1 p$ and $0 f$ ) are shown in Fig. 12. As the strength of the interaction $g$ increases (or, equivalently, the spacing between the oscillator shells diminishes), the two maxima observed in the $0 p$-shell calculation are differently affected. These effects stem from the interferences from other shells, either constructive or destructive. In short, the di-neutron configuration stands firm while the cigar-like configuration subsides. The probability of di-neutron configuration increases as $\xi$ augments until $\xi$ becomes very large $(\gg 100)$. At that point, the di-neutron probability drops from its maximum value of about $70 \%$ because higher shells in the valence space become more involved. As a result small peaks emerge, originating from higher shells and arranged in concentric rings at bigger radii, a phenomenon due to the behavior of the correlation functions $\mathcal{C}_{\alpha}(r, R)$ dis- 


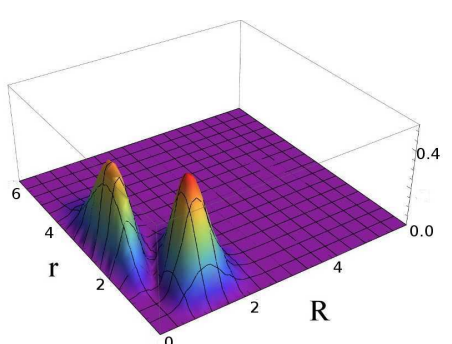

(a) $\xi=1$

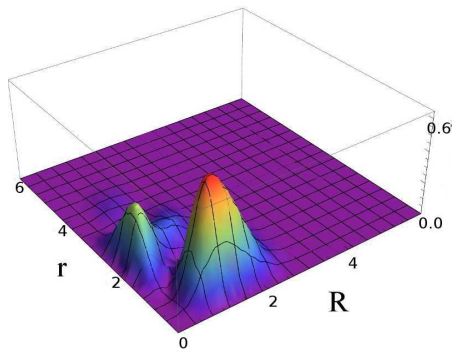

(d) $\xi=30$

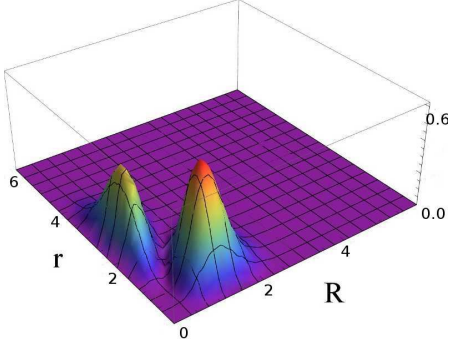

(b) $\xi=5$

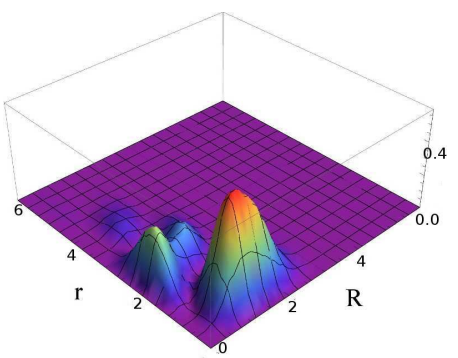

(e) $\xi=80$

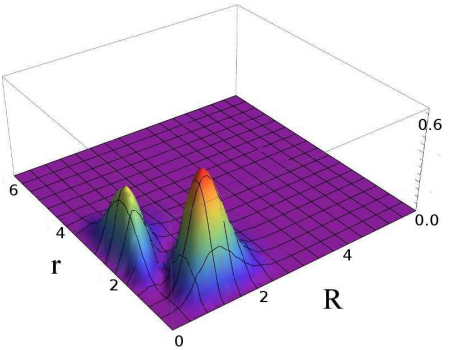

(c) $\xi=10$

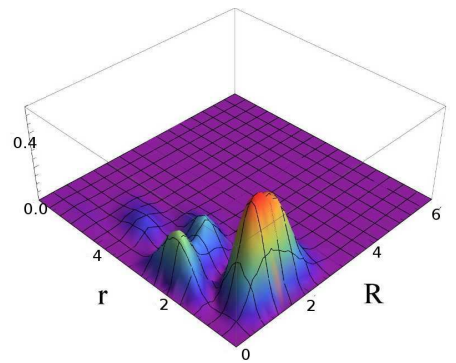

(f) $\xi=1000$

Fig. 12. The ground-state correlation function $\mathcal{C}_{0_{\mathrm{gs}}^{+}}(r, R)$ for various values of the parameter $\xi$, in the valence space containing the major oscillator shells with $1 \leq N \leq 3$.

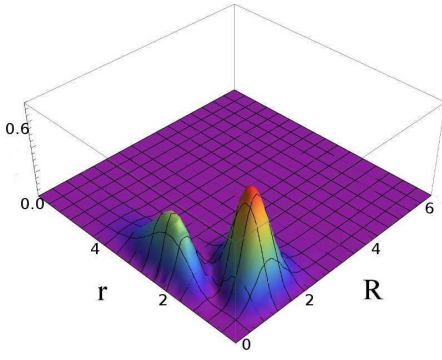

(a) $\xi=5$

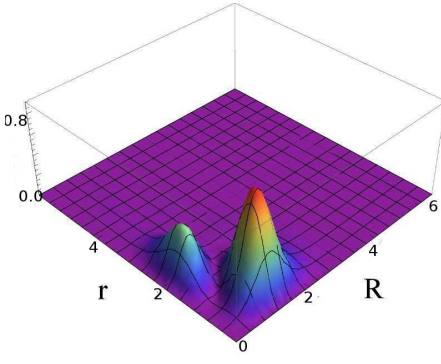

(b) $\xi=10$

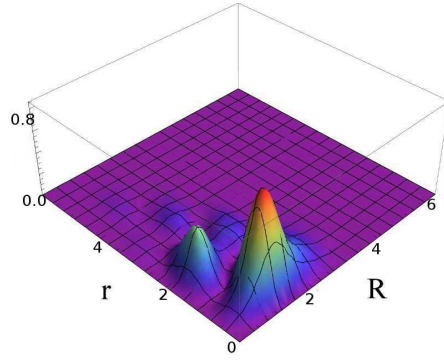

(c) $\xi=100$

Fig. 13. The ground-state correlation function $\mathcal{C}_{0_{\mathrm{gs}}^{+}}(r, R)$ for various values of the parameter $\xi$, in the valence space containing the major oscillator shells with $1 \leq N \leq 4$.

cussed in Sect. 3. Contrastingly, the probability of the cigar-like configuration diminishes continually with increasing $\xi$. In both configurations the relative distance between the two particles also alters perceivably with increasing $\xi$. The short-range attractive interaction "draws" the two neutrons closer towards each other and reduces the size of the neutron pair.

Similar conclusions can be drawn if the next major shell, $N=4$, is included in the valence space (see Fig. 13). As for the effect of the valence-space dimension, the ground state and hence its correlation function are more sensitive to the variation of $\xi$ in bigger valence spaces than they are in smaller ones. For example, in the valence space $N \leq 4$, a given value of $\xi$, say 5 , brings about a stronger shrinkage of the cigar-like configuration than it does for $N \leq 3$ [compare Figs. 12(b) and 13(a)]. 


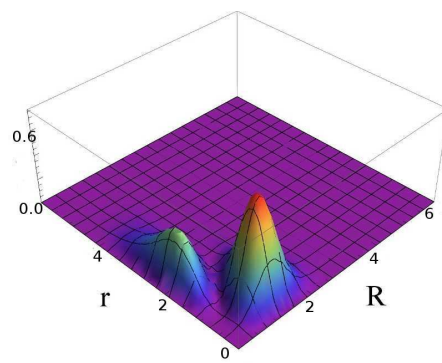

(a) $\xi=5$

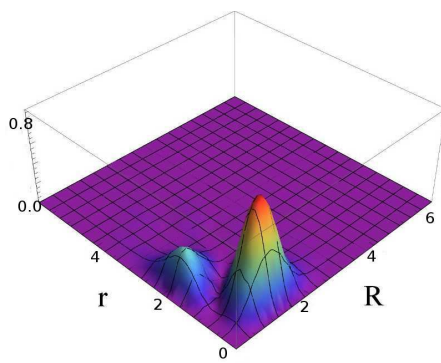

(b) $\xi=10$

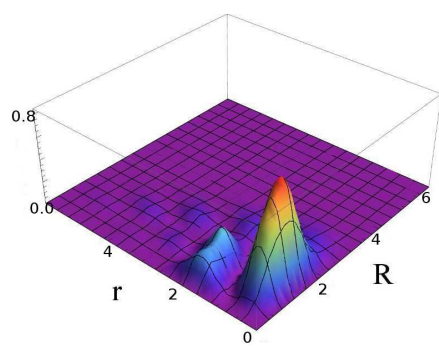

(c) $\xi=100$

Fig. 14. The ground-state correlation function $\mathcal{C}_{0_{\mathrm{gs}}^{+}}(r, R)$ for various values of the parameter $\xi$, in the valence space containing the major oscillator shells with $1 \leq N \leq 5$.

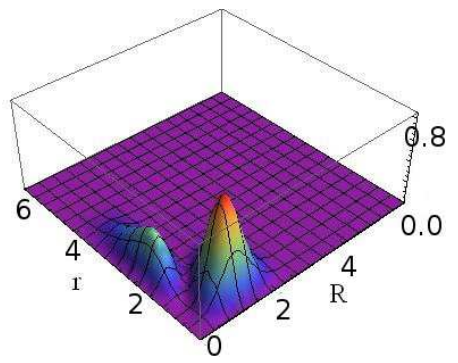

(a) $\xi=5$

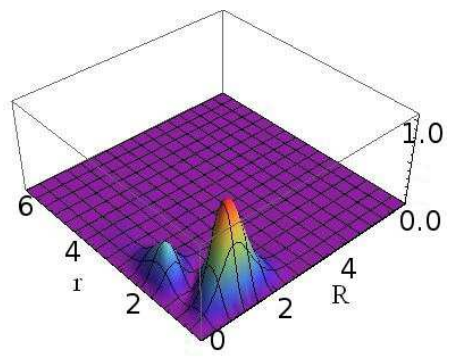

(b) $\xi=10$

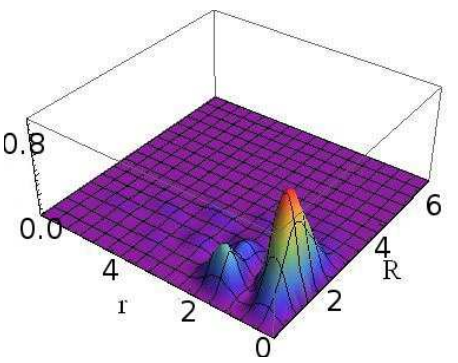

(c) $\xi=100$

Fig. 15. The ground-state correlation function $\mathcal{C}_{0_{\mathrm{gs}}^{+}}(r, R)$ for various values of the parameter $\xi$, in the valence space containing the major oscillator shells with $1 \leq N \leq 6$.

As the valence space is further expanded, the evolution of the ground-state correlation function maintains its major features (see Fig. 14 for the valence space with $1 \leq N \leq 5)$. With expanding valence space the maximum of the di-neutron configuration grows more pronounced. The results obtained so far indicate that the effect of the configuration interaction does not strongly depend on the size of the valence space into which the two neutrons are excited from the $0 p$ shell. This is further supported by the calculation carried out in the valence space with $1 \leq N \leq 6$ shown in Fig. 15 .

\subsection{Elimination of the center-of-mass motion}

The assumption in the shell model of a fixed origin is in violation of translational invariance that is a requisite for a nuclear Hamiltonian. If particles are allowed to occupy more than one major oscillator shell, spurious states may occur as a result. The results of the preceding subsection, therefore, should be taken with care since spurious admixtures have not been eliminated.

A convenient way of dealing with the elimination of the center-of-mass motion, is by adopting the cluster-orbital shell model (COSM) [18-20], which unifies the shell model and the cluster model. The model is well suited for the present 
purpose since it is specifically designed to treat halo nuclei. In addition, it has the advantage that it is free from spurious center-of-mass motion, which is achieved by introducing translationally invariant coordinates between the nucleons and the center-of-mass of the core. No description is given here of COSM for which the reader is referred to the original papers by Suzuki et al. [18-20], whose notation will be closely adhered to in the following.

\subsection{The two-particle correlation function in ${ }^{6} \mathrm{He}$}

Suzuki et al. have studied the helium isotopes in the strictly formulated COSM in Refs. [19,20]. In this subsection the results of this work are used to explore two-particle spatial correlations in ${ }^{6} \mathrm{He}$.

The schematic Hamiltonian (31) is modified in COSM and acquires the form

$$
\hat{H}^{\prime}=\sum_{i=1}^{2}\left(\frac{1}{2 \mu} p_{i}^{2}+\frac{1}{2} \mu \omega^{2} r_{i}^{2}\right)-g \delta\left(\bar{r}_{1}-\bar{r}_{2}\right)+\frac{1}{(f+1) \mu} \bar{p}_{1} \cdot \bar{p}_{2},
$$

where $f$ is the number of nucleons in the core $\left(f=4\right.$ for $\left.{ }^{6} \mathrm{He}\right)$ and $\mu$ is the reduced mass, $\mu=m f /(f+1)$. The correlation functions in the various valence spaces are to be re-evaluated with the Hamiltonian (32). In addition, an estimate of $\xi=g b^{-3} / \hbar \omega$ (see Subsect. 4.3) is needed in the case of ${ }^{6} \mathrm{He}$. This parameter depends on the oscillator length $b$ and the strength of the delta interaction $g$. The spacing between two consecutive harmonic-oscillator levels, $\hbar \omega$, can be deduced from $b$ through $\hbar \omega=\hbar^{2} / \mu b^{2}$.

An estimate of the oscillator length $b$ can be given as follows. Within the framework of COSM, the following relation is valid between the matter radii of ${ }^{4} \mathrm{He}$ and ${ }^{6} \mathrm{He}$ :

$$
6 R_{\mathrm{m}}^{2}\left({ }^{6} \mathrm{He}\right)=\frac{5}{6}\left\langle x_{1}^{2}+x_{2}^{2}\right\rangle-\frac{1}{3}\left\langle\bar{x}_{1} \cdot \bar{x}_{2}\right\rangle+4 R_{\mathrm{m}}^{2}\left({ }^{4} \mathrm{He}\right),
$$

where $\bar{x}_{i}(i=1,2)$ are the position vectors of the valence nucleons with respect to the center of mass of the core with its position vector $\bar{R}_{\mathrm{c}}$,

$$
\bar{x}_{i}=\bar{r}_{i}-\sqrt{\frac{1}{f}} \bar{R}_{\mathrm{c}}
$$

The expectation values $\left\langle x_{i}^{2}\right\rangle$ and $\left\langle\bar{x}_{1} \cdot \bar{x}_{2}\right\rangle$ are directly related to $b$ which appears in the wave function. An estimate of $b$ is obtained by assuming both neutrons to occupy the $0 p$ shell, coupled to orbital angular momentum $L=0$. It then follows that $\left\langle x_{1}^{2}\right\rangle=\left\langle x_{2}^{2}\right\rangle=\frac{5}{2} b^{2}$ and $\left\langle\bar{x}_{1} \cdot \bar{x}_{2}\right\rangle=0$, and therefore

$$
b^{2}=\frac{36}{25} R_{\mathrm{m}}^{2}\left({ }^{6} \mathrm{He}\right)-\frac{24}{25} R_{\mathrm{m}}^{2}\left({ }^{4} \mathrm{He}\right) .
$$




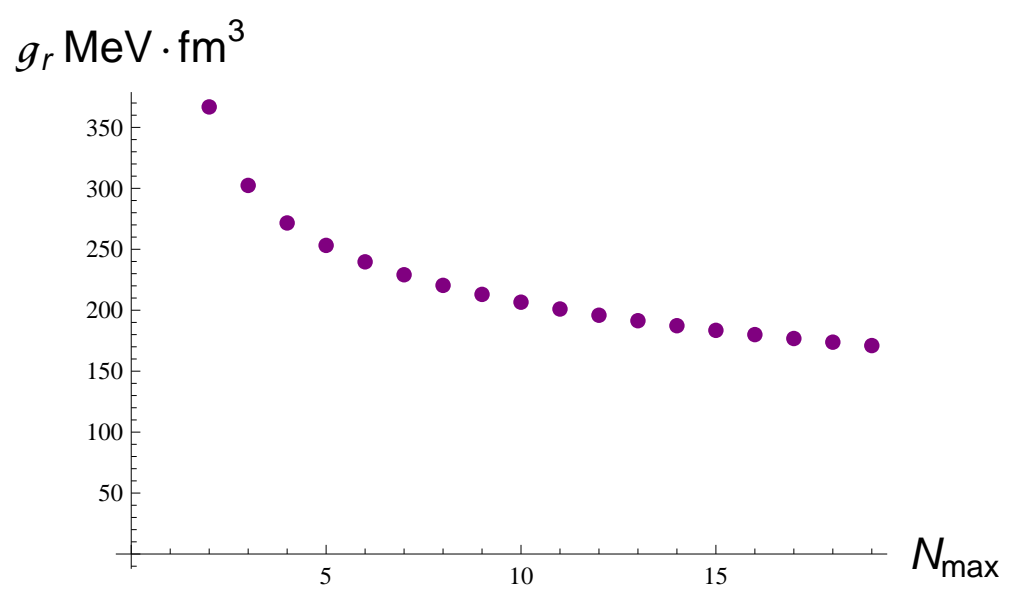

Fig. 16. The values of $g$ that reproduce the observed $0^{+}-2^{+}$energy splitting in ${ }^{6} \mathrm{He}$ as a function of the number of shells in the valence space.

The nuclear matter radius of ${ }^{6} \mathrm{He}$ was determined by proton elastic scattering in inverse kinematics $[21,22]$. The values thus obtained are somewhat smaller than those of Ref. [23] which are based on an analysis of the interaction and fragmentation cross sections combined with a Glauber-model calculation. For consistency, it is better to use in Eq. (35) matter radii obtained with the same method and therefore the values of Ozawa et al. [23] are taken, $R_{\mathrm{m}}\left({ }^{4} \mathrm{He}\right)=$ 1.57 (4) fm and $R_{\mathrm{m}}\left({ }^{6} \mathrm{He}\right)=2.48(3) \mathrm{fm}$. One finds $b=2.55 \mathrm{fm}$, which is the oscillator length adopted in the following.

Next, an estimate of the strength of the delta interaction is needed. From the schematic calculations presented in Subsect. 4.3, a dependence of $g$ on the size of the valence space is expected. In fact, it has been shown that the threedimensional delta interaction only has physical meaning in a truncated space and that for free neutrons, asymptotically, the magnitude of $g$ varies inversely with the cutoff in momentum space [24]. In the present application the strength of the delta interaction is obtained from the excitation energy of the first $2^{+}$ state which is observed in ${ }^{6} \mathrm{He}$ as a resonance at 1.797 (25) $\mathrm{MeV}$ [25]. For a given valence-space truncation in the principal oscillator quantum number $N$, a value of $g$ is adopted which reproduces this $0^{+}-2^{+}$energy splitting. This procedure, for a given valence space, completely fixes the parameter $\xi$, and hence the structure of the ground state of ${ }^{6} \mathrm{He}$ and the two-particle correlations in the ground state. The values of $g$ that reproduce the observed $0^{+}-2^{+}$energy splitting are plotted in Fig. 16 as a function of the valence space defined by $1 \leq N \leq N_{\max }$ with $N_{\max }$ varying from 2 to 19 . As expected, $g$ decreases as the valence space expands.

As a consistency check on the values thus obtained for the interaction strength $g$, an estimate can be made of the two-neutron separation energy $S_{2 n}$. The single-particle energy of the $0 p$ shell relative to the ${ }^{4} \mathrm{He}$ core can be defined phenomenologically and should be equal to $-S_{\mathrm{n}}$, where $S_{\mathrm{n}}=-0.89(5) \mathrm{MeV}$ is 


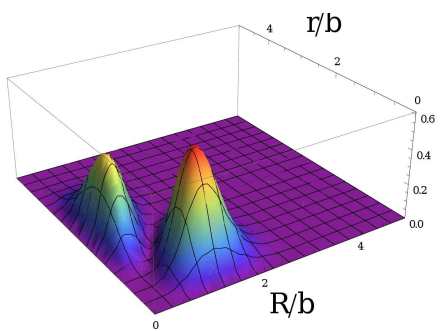

(a) $1 \leq N \leq 3$

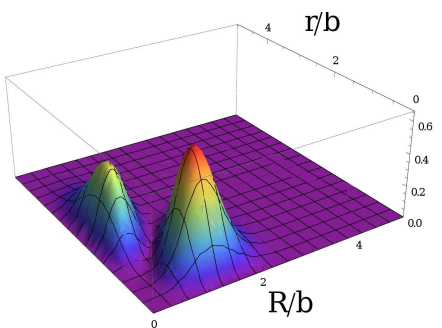

(b) $1 \leq N \leq 4$

Fig. 17. The correlation function $\mathcal{C}_{0_{\mathrm{gs}}^{+}}(r, R)$ as obtained in two different valence spaces with $N_{\max }=3$ and 4 , respectively, and with the strength $g$ adjusted to the observed $0^{+}-2^{+}$energy splitting in ${ }^{6} \mathrm{He}$.

the neutron separation energy in ${ }^{5} \mathrm{He}$ [26]. Once the valence-space truncation is fixed, so is $S_{2 \mathrm{n}}$ in ${ }^{6} \mathrm{He}$. For $N_{\max }=2$ and 3, one finds $S_{2 \mathrm{n}}=0.91 \mathrm{MeV}$ and $S_{2 \mathrm{n}}=0.81 \mathrm{MeV}$, respectively. These results are in reasonable agreement with the measured value of $S_{2 \mathrm{n}}=0.972$ (1) $\mathrm{MeV}$ for the ground state of ${ }^{6} \mathrm{He}[26]$.

The wave functions of the $0^{+}$ground state in the various valence spaces are stable, with $\sim 92 \%$ probability of being in the state $\left|(0 p)^{2} ; 00\right\rangle$ followed by $\sim 4 \%$ probability of being in $\left|(0 d)^{2} ; 00\right\rangle$. Other components are negligibly small. In consequence, the correlation function $\mathcal{C}_{0_{\mathrm{gs}}^{+}}(r, R)$ is determined by the two major components arising from the $0 p$ and $0 d$ shells, and is very insensitive to the size of the valence space as long as the latter contains the $N=2$ major shell (to include the $0 d$ shell). This is illustrated in Fig. 17 for the valence spaces $1 \leq N \leq 3$ with $g=302 \mathrm{MeV} \mathrm{fm}^{3}$ and $1 \leq N \leq 4$ with $g=272 \mathrm{MeV} \mathrm{fm}^{3}$. The probability of the di-neutron configuration amounts to $60 \%$ with the first truncation and $61 \%$ with the second. The ground-state wave function has as principal component $\left|(0 p)^{2} ; 00\right\rangle$ with $\left|(0 d)^{2} ; 00\right\rangle$ being a much smaller one. This result is not influenced appreciably by the size of the valence space as long as it contains the $0 d$ shell $\left(N_{\max } \geq 2\right)$. This finding is consistent with ${ }^{6} \mathrm{He}$ being a halo nucleus because of the argument that halo nucleons can only exist in $s$ and $p$ waves [2].

\section{Conclusion}

The main result of this paper can be summarized as follows. The existence of the two well-known geometric configurations of ${ }^{6} \mathrm{He}$, commonly referred to as di-neutron and cigar-like, is shown to be an inescapable consequence of the geometry of the $0 p$ shell. The simple fact of placing the two outer neutrons in the $0 p$ shell and of imposing antisymmetry, determines many of the properties of the spatial correlations of ${ }^{6} \mathrm{He}$ and, in particular, it leads to the equiprobability of the di-neutron and cigar-like configurations in the ground state of ${ }^{6} \mathrm{He}$. Only if the neutrons are scattered into higher shells can 
the reflection-like symmetry with respect to the $r=2 R$ plane be broken and this equiprobability of the two configurations be lifted. Furthermore, any reasonable choice of residual interaction suppresses the cigar-like and enhances the di-neutron configuration which therefore becomes dominant in the ground state.

To a large extent, the validity of these results has been acknowledged in a variety of ways by the nuclear physics community interested in halo phenomena [4]. The results presented in this paper show that no complicated calculations in large model spaces are needed to arrive at these conclusions but that they can be obtained in an elementary version of the shell model. In fact, much of the information on spatial two-particle correlations is contained in the angular correlation function for which an analytic expression is available as a sum of Legendre polynomials in the cosine of the angle between the position vectors of the particles. It will transpire that angular correlation functions are well adapted for the purpose of establishing a geometry of systems with four particles, which is the topic of part II of this series.

\section{Acknowledgment}

We wish to thank Navin Alahari, Kris Heyde and Peter Schuck for fruitful discussions at various stages of this work. This work was partially supported by the Agence Nationale de Recherche, France, under contract nr ANR-07BLAN-0256-03.

\section{References}

[1] I. Tanihata, H. Hamagaki, O. Hashimoto, Y. Shida, N. Yoshikawa, K. Sugimoto, O. Yamakawa, T. Kobayashi and N. Takahashi, Phys. Rev. Lett. 55 (1985) 2676.

[2] A.S. Jensen, K. Riisager, D.V. Fedorov and E. Garrido, Rev. Mod. Phys. 76 (2004) 215.

[3] I. Tanihata, D. Hirata, T. Kobayashi, S. Shirnoura, K. Sugimoto and H. Toki, Phys. Lett. B 289 (1992) 261.

[4] M.V. Zhukov, B.V. Danilin, D.V. Fedorov, J.M. Bang, I.J. Thompson and J.S. Vaagen, Phys. Reports 231 (1993) 151.

[5] M.G. Mayer, Phys. Rev. 75 (1949) 1969.

[6] J.H.D. Jensen, H. Suess and O. Haxel, Die Naturwissenschaften 36 (1949) 155.

[7] B.A. Brown and W.A. Richter, Phys. Rev. C 74 (2006) 034315. 
[8] M. Honma, T. Otsuka, B.A. Brown and T. Mizusaki, Phys. Rev. C 69 (2004) 034335 .

[9] D.H. Wilkinson, Annu. Rev. Nucl. Part. Sci. 45 (1995) 1.

[10] I. Talmi, Helv. Phys. Acta 25, 185 (1952)

[11] M. Moshinsky, Nucl. Phys. 13, 104 (1959)

[12] M. Moshinsky and Yu.F. Smirnov, The Harmonic Oscillator in Modern Physics. (Harwood. Academic Publishers, Amsterdam, 1996).

[13] I. Talmi, Simple Models of Complex Nuclei. The Shell Model and Interacting Boson Model, Harwood, Chur, 1993.

[14] A. Barber and B.S. Cooper, Nucl. Data Tables 10 (1971) 49.

[15] F. Catara and A. Insolia, Phys. Rev. C 29 (1984) 3.

[16] N. Pillet, N. Sandulescu and P. Schuck, Phys. Rev. C 76 (2007) 024310.

[17] S. Cohen and D. Kurath, Nucl. Phys. A 101 (1967) 1.

[18] Y. Suzuki and K. Ikeda, Phys. Rev. C 38 (1988) 410.

[19] Y. Suzuki and J.J. Wang, Phys. Rev. C 41 (1990) 736.

[20] Y. Suzuki, Nucl. Phys. A 528 (1991) 395.

[21] G.D. Alkhazov et al., Phys. Rev. Lett. 78 (1997) 2313.

[22] O.A. Kiselev et al., Eur. Phys. J. A 25, Suppl. 1 (2005) 215.

[23] A. Ozawa, T. Suzuki and I. Tanihata, Nucl. Phys. A 693 (2001) 32.

[24] H. Esbensen, G.F. Bertsch and K. Hencken, Phys. Rev. C 56 (1997) 3054.

[25] D.R. Tilley, C.M. Cheves, J.L. Godwin, G.M. Haled, H.M. Hofmann, J.H. Kelley, C.G. Sheua and H.R. Weller, Nucl. Phys. A 708 (2002) 3.

[26] G. Audi, A. H. Wapstra, and C. Thibault, Nucl. Phys. A 729 (2003) 337. 